\title{
COCRISTAIS: UMA ESTRATÉGIA PROMISSORA NA ÁREA FARMACÊUTICA
}

\author{
Alanny B. O. Rocha ${ }^{a}$, Gislaine Kuminek ${ }^{a}$, Tatiane C. Machado ${ }^{a}$, Juliana Rosa ${ }^{a}$, Gabriela S. Rauber ${ }^{\text {de }}$, Paola A. A. Borba ${ }^{a}$, \\ Sana Siedler ${ }^{\text {, }}$ Hellen K. Stulzera , Silvia Lucia Cuffini ${ }^{\mathrm{b}}$, Naír Rodríguez-Hornedo ${ }^{\mathrm{c}, \mathrm{e}}$ e Simone G. Cardoso ${ }^{\mathrm{a}, *}$ \\ a Departamento de Ciências Farmacêuticas, Universidade Federal de Santa Catarina, 88040-970 Florianópolis - SC, Brasil \\ ${ }^{\mathrm{b}}$ Instituto de Ciências e Tecnologia, Universidade Federal de São Paulo, 12231-280 São José dos Campos - SP, Brasil \\ 'Department of Pharmaceutical Sciences, University of Michigan, 48109 Ann Arbor - MI, United States \\ ${ }^{\mathrm{d}}$ Department of Chemistry, University of Cambridge, CB2 1EW Cambridge - Cambridgeshire, United Kingdom

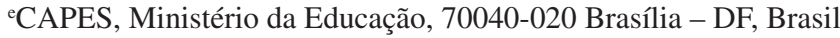

Recebido em 05/02/2016; aceito em 12/05/2016; publicado na web em 05/08/2016

\begin{abstract}
COCRYSTALS: A PROMISING STRATEGY IN PHARMACEUTICAL SCIENCES. Pharmaceutical cocrystals have emerged as a useful strategy to improve the aqueous solubility of poorly water soluble drugs by aiming to enhance their oral absorption and bioavailability. Aqueous cocrystal solubility can be orders of magnitude higher than that of the constituent drug and this solubility advantage can be fine-tuned based on environmental conditions such as $\mathrm{pH}$ and the presence of drug solubilizing agents. This review presents a brief overview of pharmaceutical cocrystals regarding cocrystal design, obtainment methods, with particular focus on cocrystal solubility, and the solubility modulation by solution phase chemistry.
\end{abstract}

Keywords: cocrystals; drug; solubility; transition point; thermodynamic stability.

\section{INTRODUÇÃO}

O setor de fármaco-medicamentos representa uma área estratégica sob o ponto de vista tecnológico/produtivo nacional. Vários são os desafios da produção de medicamentos competitivos e de qualidade no Brasil, como a oferta de insumos, a pesquisa de novas moléculas ativas e a sua inserção no mercado. Neste sentido, a utilização de inovações incrementais surge como alternativa à problemática do setor, destacando-se a manipulação da forma cristalina dos fármacos, síntese de polimorfos, sais, complexos e, mais recentemente, os cocristais. ${ }^{1}$

Cocristais são materiais cristalinos homogêneos, compostos por dois ou mais compostos em proporção estequiométrica definida e que são sólidos em condições ambientes $\left(25^{\circ} \mathrm{C} / 1\right.$ atm $) .{ }^{2,3}$ Ao longo das últimas décadas, os cocristais têm recebido atenção significativa por parte da indústria farmacêutica, e diversos cocristais farmacêuticos têm sido relatados. Cocristais farmacêuticos, por sua vez, são compostos por um ingrediente farmacêutico ativo (IFA) e uma molécula não tóxica ou outro IFA, chamado de coformador. Ligações de hidrogênio entre as moléculas neutras do fármaco e do coformador orientam a formação do cocristal. ${ }^{2,4}$

A síntese de cocristais tem sido uma estratégia cada vez mais utilizada para melhorar a solubilidade, a taxa de dissolução in vitro, e consequentemente, a biodisponibilidade de fármacos pouco solúveis, sem a necessidade de mudar a estrutura molecular e/ou a interação farmacológica. ${ }^{5-7}$ No entanto, a avaliação da solubilidade e estabilidade dos cocristais em solução ainda não está consolidada. Um levantamento realizado no período de 1999 a 2015 (Figura 1) revelou um crescente número de publicações envolvendo a síntese e a caracterização dos cocristais, mas um reduzido número de publicações envolvendo outros aspectos essenciais no desenvolvimento de formulações contendo cocristais, como estudos de solubilidade, química em solução, estabilidade e a influência de excipientes.

Considerando que a baixa solubilidade aquosa é um dos principais problemas associados às entidades químicas farmacêuticas, os

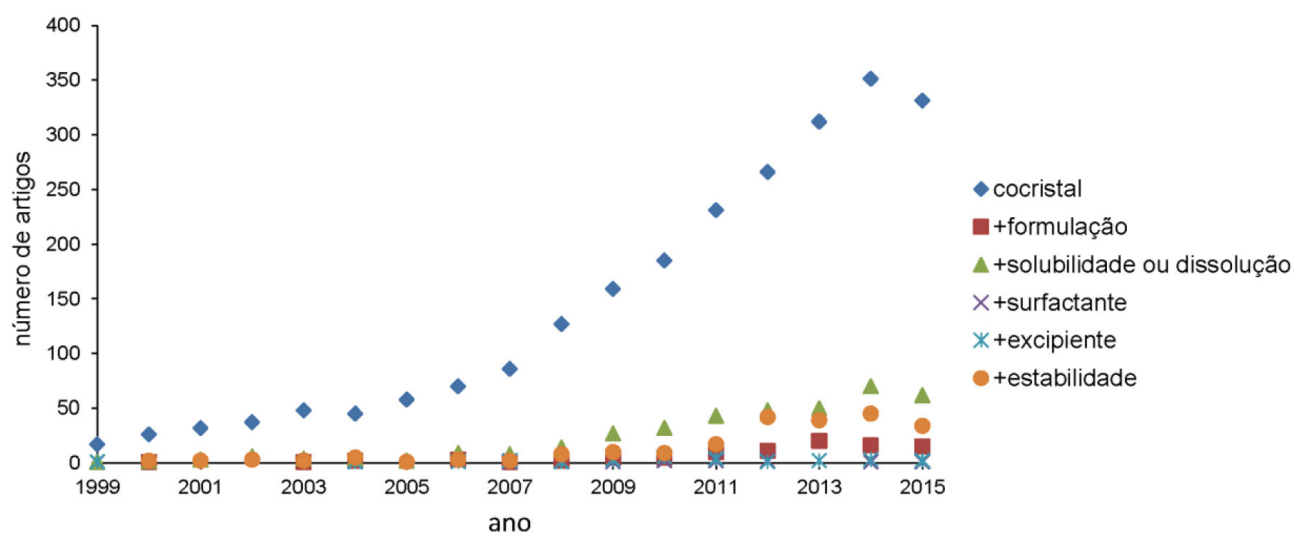

Figura 1. Número de artigos publicados por ano com o tema cocristais, indicando maior número de publicações abrangendo formação de cocristais em comparação com as publicações relacionadas às propriedades farmacêuticas. Dados obtidos da base de dados Scopus considerando as revistas farmacêuticas, químicas, e das engenharias de materias e química, até o final do ano de 2015. O símbolo + representa a busca de cocristal somado à propriedade indicada 
cocristais tornam-se promissores por apresentarem solubilidade até 100 vezes superior em relação ao fármaco no estado cristalino, ao passo que outras formas sólidas, como polimorfos e solvatos, não são capazes de alcançar tamanha dimensão (Figura 2). ${ }^{4}$

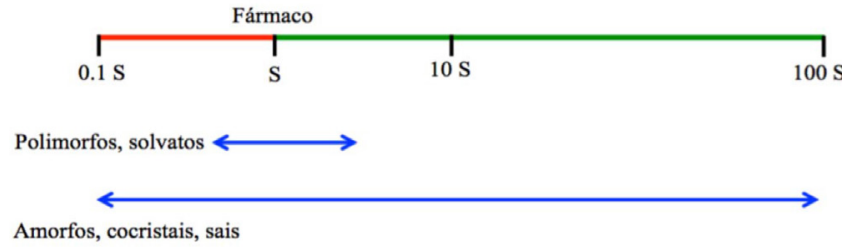

Figura 2. Esquema comparativo de diferentes formas cristalinas de um mesmo fármaco, incluindo forma amorfa, e as variadas extensões de solubilidade que estas podem apresentar

Tem sido demonstrado que cocristais formados por coformadores que sejam pelo menos 10 vezes mais solúveis do que o fármaco terão sua solubilidade aumentada. ${ }^{4}$ Ainda, este aumento parece ser diretamente proporcional à razão das solubilidades do coformador e do fármaco, como apresentado na Figura 3, atuando como um índice de supersaturação, permitindo, assim, orientar a seleção e a formulação do cocristal. ${ }^{8}$

Vale ressaltar, no entanto, que um cocristal altamente solúvel nem sempre é desejado, uma vez que esta vantagem da solubilidade ( solubility advantage, SA) pode não ser mantida durante a dissolução do cocristal. Assim como outros sistemas que geram supersaturação, os cocristais são passíveis de conversão à forma sólida mais estável quando em contato com soluções. ${ }^{9}$ Neste caso, a adição de agentes solubilizantes do fármaco, para diminuir a vantagem da solubilidade, bem como de inibidores de cristalização, podem ser introduzidos ao meio ou à formulação a fim de evitar ou reduzir as conversões. ${ }^{8}$

Outro fator interessante é que a solubilidade dos cocristais pode ser modulada de modo que fique dentro ou fora da região de estabilidade dos cocristais, dependendo da composição do meio como $\mathrm{pH},{ }^{10-13}$ presença de aditivos, ${ }^{14-17}$ concentração de coformador, ${ }^{18}$ entre outras.

A possibilidade de modulação da solubilidade dos cocristais é atribuída às propriedades físico-químicas dos coformadores e às interações moleculares dos componentes dos cocristais em solução (complexação, ionização e solubilização micelar). ${ }^{9,19}$ A simples mudança do $\mathrm{pH}$ da solução pode ser responsável por manter a estabilidade termodinâmica ou a vantagem da solubilidade do sistema. Como pode ser observado na Figura 4, o comportamento da curva de solubilidade de cocristais formados por coformadores com características variadas difere da curva de solubilidade do fármaco. Em alguns valores de $\mathrm{pH}$ os cocristais podem ser até 1000 vezes mais solúveis do que o fármaco, ao passo que em outros valores de $\mathrm{pH}$ o cocristal pode apresentar-se menos solúvel. ${ }^{10}$

Apesar disso, diversos estudos de solubilidade e dissolução de cocristais encontrados na literatura desconsideram a enorme alteração que o $\mathrm{pH}$ e a presença de aditivos e excipientes podem ocasionar nestes ensaios. Em outros casos, os estudos são realizados em condições que não mimetizam o meio existente in vivo e, assim, correlações in vivo/in vitro são escassas.

Embora haja um pequeno número de publicações no Brasil, o interesse pelo tema é crescente e vem sendo abordado em distintos eventos, dissertações e teses no país. Desta forma, o presente trabalho buscou abordar os principais aspectos relacionados aos cocristais farmacêuticos, visando contribuir para o avanço do tema no país, através de uma revisão recente e completa da literatura atual destacando, principalmente, a possibilidade de modular a solubilidade e a estabilidade destes sólidos.

\section{DESENHO DO COCRISTAL}

A engenharia de cristais correlaciona interações intermoleculares, estruturas cristalinas e propriedades, aplicando conceitos de química supramolecular ao desenho de materiais cristalinos. Este termo foi adotado primeiramente por Pepinsky, em 1955, e difundido por Schmidt. Entretanto, foi somente com o advento dos estudos envolvendo a formação de cocristais que a área entrou em ascensão. ${ }^{20}$ Isso se deve a dois fatores principais: (i) a possibilidade de desenhar e estudar fenômenos de química supramolecular em experimentos de cocristalização, e (ii) a aplicabilidade dos materiais desenvolvidos, por exemplo na indústria farmacêutica. ${ }^{20,21}$

Com relação aos cocristais farmacêuticos, na grande maioria das vezes os coformadores são selecionados pela presença de regiões de complementaridade molecular com o fármaco, especialmente pela

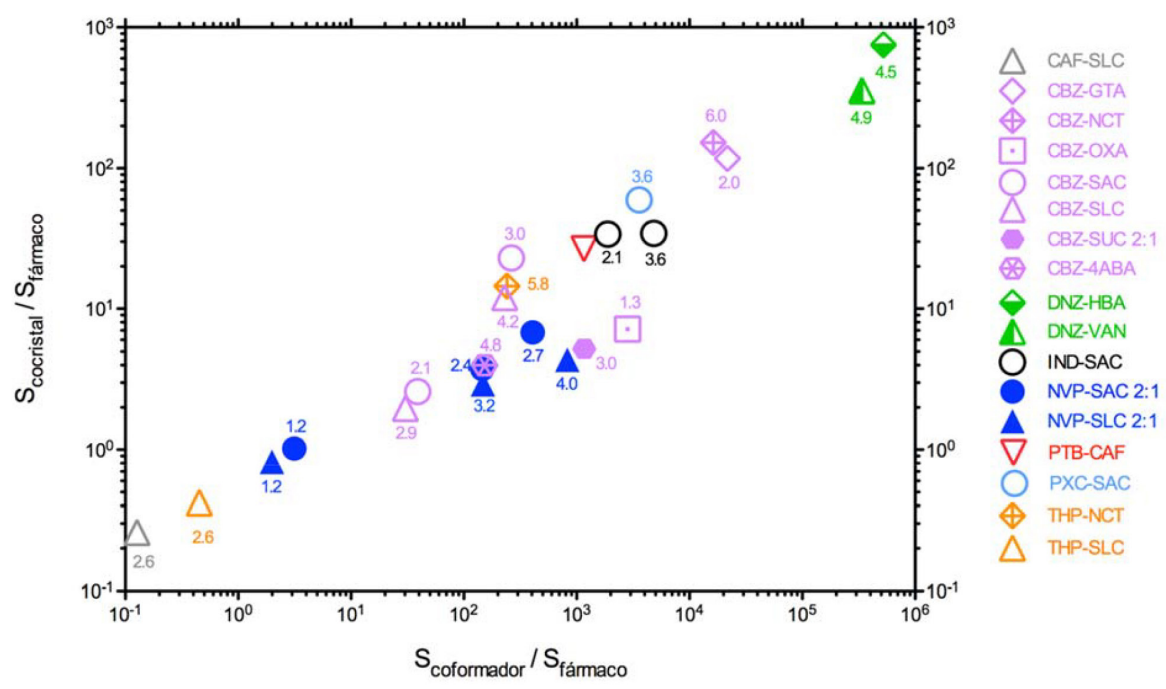

Figura 3. Vantagem da solubilidade do cocristal sobre o fármaco $\left(S A=S_{\text {cocristal }} / S_{\text {fármaco }}\right)$ versus a razão da solubilidade entre coformador e fármaco $\left(S_{\text {coformador }} /\right.$ $\left.S_{\text {fármaco }}\right) . S_{\text {coformado }} / S_{\text {fármaco }}>10$ leva a uma $S_{\text {cocristal }} / S_{\text {fármaco }}>1$. Cafeína $(C A F)$, ácido salicílico (SLC), carbamazepina (CBZ), ácido glutárico (GTA), nicotinamida (NCT), ácido oxálico (OXA), sacarina (SAC), ácido succínico (SUC), ácido 4-aminobenzóico (4ABA), danazol (DNZ), ácido hidróxibenzóico (HBA), vanilina (VAN), indometacina (IND), nevirapina (NVP), ácido maleico (MLE), pteroestilbeno (PTB), piroxicam (PXC), teofilina (THP). Adaptada de Kuminek et al. (2016), ${ }^{8}$ com permissão da Elsevier 

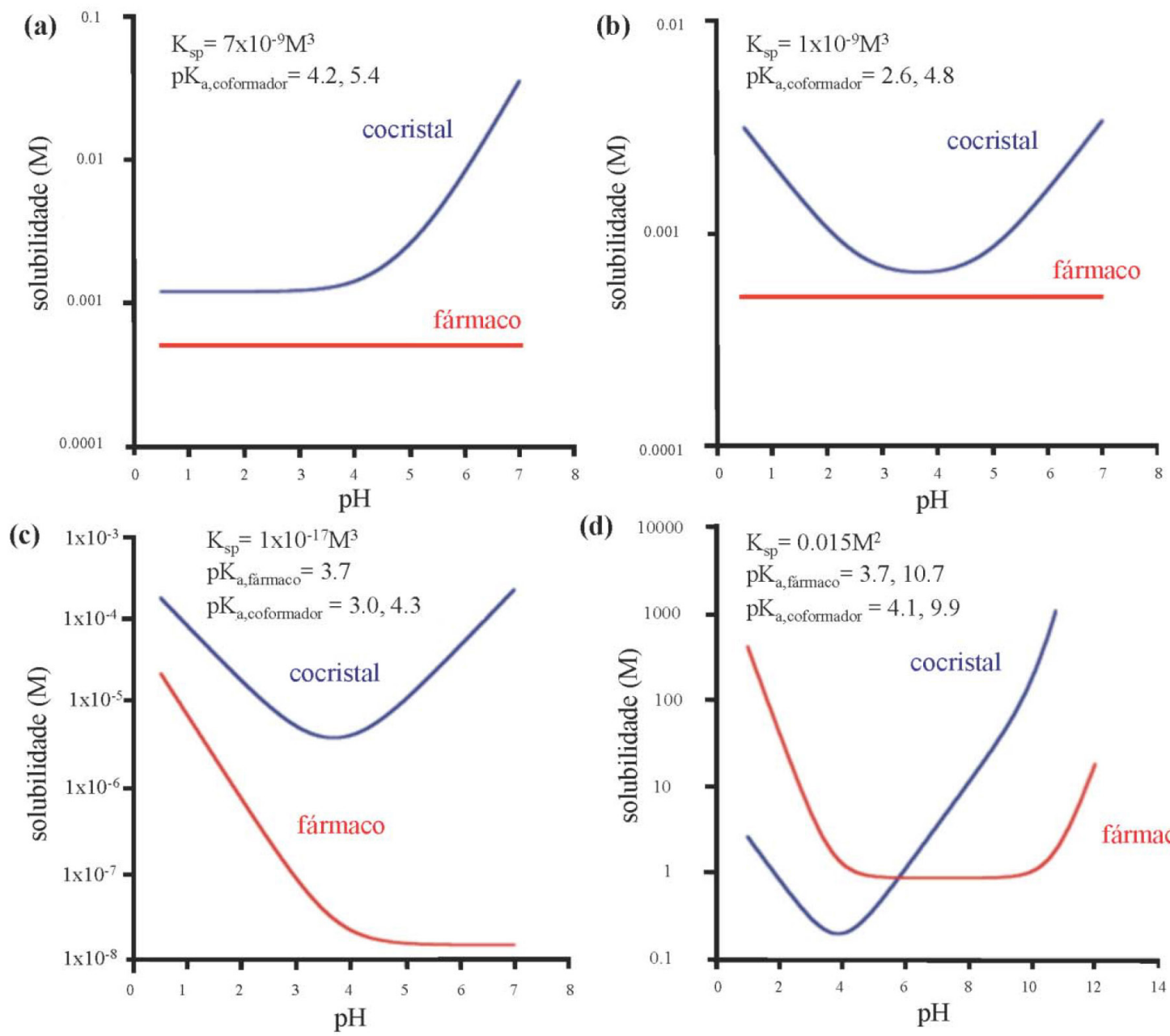

(d)

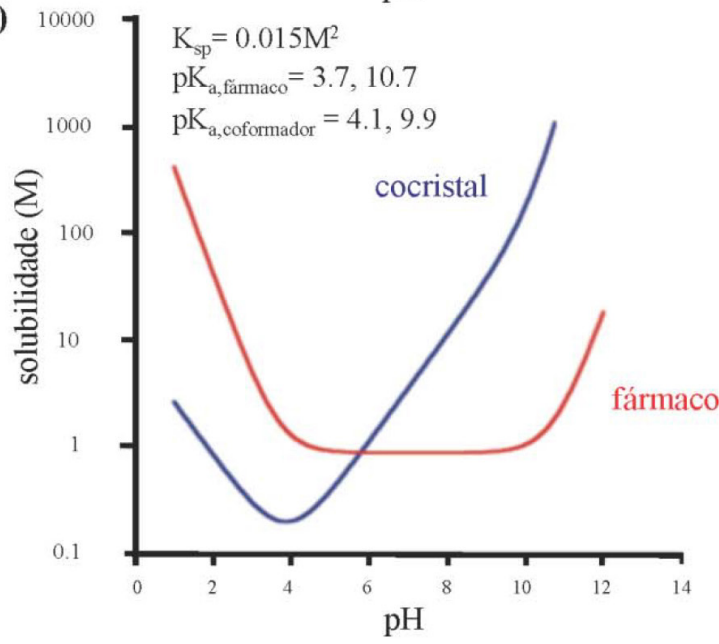

Figura 4. Perfis de solubilidade em função do pH para (a) cocristal 2:1 $R_{2} H_{2} A$, fármaco não ionizável (R) e coformador ácido diprótico ( $\left.H_{2} A\right)(b)$ cocristal $2: 1$ $R_{2} H A B$, fármaco não ionizável $(R)$ e coformador anfotérico $(H A B)(c)$ cocristal $2: 1 B_{2} H_{2} A$, fármaco básico $(B)$ e coformador ácido diprótico $\left(H_{2} A\right)$, $e(d)$ cocristal 1:1 $-\mathrm{ABH} \mathrm{H}^{+} \mathrm{H}_{2} \mathrm{X}$, fármaco zwiteriônico $\left(-\mathrm{ABH}^{+}\right)$e coformador ácido diprótico $\left(\mathrm{H}_{2} \mathrm{X}\right)$. Os valores de $\mathrm{p} K_{a}$ para o fármaco e coformador, e o $K_{s p}$ estão descritos em cada gráfico. Os valores de $K_{s p}$ foram determinados experimentalmente ou estimados a partir de trabalhos já publicados para cada cocristal selecionado: (a) carbamazepina-ácido-succínico, (b) carbamazepina-ácido-4-aminobenzóico, (c) itraconazol-ácido-L-tartárico e (d) gabapentina-ácido-3-hidroxibenzóico. "Reproduzida com permissão de Bethune et al., 2009. ${ }^{10}$ Copyright 2009 American Chemical Society"

presença de grupos funcionais capazes de formar ligações de hidrogênio intermoleculares. De fato, análises utilizando a plataforma $\mathrm{CSD}^{22}$ (Cambridge Structural Database) revelam que o modo básico de interação fármaco:coformador nos cocristais compreende as ligações de hidrogênio. Entretanto, interações de Van der Waals, empilhamento $\pi$ e ligações de halogênio também se demonstram significativas para a formação dos cocristais. Além disto, os coformadores adequados para uso farmacêutico devem apresentar-se seguros em relação à sua toxicidade. Neste sentido, existem mais de uma centena de materiais sólidos reconhecidos como seguros pelo GRAS (Generally Recognized as Safe) do Food and Drug Administration (FDA). Nesta lista destacam-se aditivos alimentares e outras substâncias já bastante estudadas. Entretanto, quantidades subterapêuticas de fármacos como ácido acetilsalicílico e paracetamol também podem ser utilizadas como coformadores. A Figura 5 representa uma relação de alguns dos coformadores mais frequentemente descritos na literatura (CSD, patentes e trabalhos acadêmicos). ${ }^{23,24}$

Um dos conceitos mais utilizados no desenho de sólidos cristalinos é o de synthons supramoleculares. Synthons são classificados como unidades subestruturais críticas que contém um máximo de informação em padrão de tamanho reduzido e suficientemente estáveis para serem consideradas entidades independentes. ${ }^{25,26}$ Quimicamente falando, são padrões de interações não covalentes que se repetem entre as moléculas e contribuem para a estruturação tridimensional de um sólido, no caso, um cocristal. Synthons formados por interações entre dois grupos funcionais iguais são classificados como homosynthons, enquanto que as interações entre dois grupos funcionais diferentes são mais favoráveis e caracterizam os heterosynthons (Figura 6). ${ }^{27}$

Além de interações baseadas em complementaridade molecular, cabe ressaltar que outros fenômenos podem estar envolvidos na formação de um cocristal. O empacotamento, a estereoquímica dos componentes e características intrínsecas do fármaco, como a polaridade e o $\mathrm{pK}_{\mathrm{a}}$, também devem ser considerados durante a seleção do coformador para a síntese do cocristal. $\mathrm{O} \Delta \mathrm{pK}_{\mathrm{a}}$ entre fármaco e coformador é um dos parâmetros utilizados. Como regra geral, para reações em solução, $\Delta \mathrm{pK}_{\mathrm{a}}<0$ indica a formação de um cocristal, enquanto que $\Delta \mathrm{pK}_{\mathrm{a}}>3$ tende a resultar em um sal. No caso da região de $\Delta \mathrm{pK}_{\mathrm{a}}$ entre 0 e 3 , a habilidade de prever o material resultante é limitada. ${ }^{28,29}$ Desta forma, este critério de avaliação baseado no $\mathrm{pK}_{\mathrm{a}}$ é apenas um guia no desenho de cocristais, visto que não é aplicável em todas as situações (ex.: itraconazol:ácido succínico $2: 1)^{6}$ e pode apresentar diferenças de acordo com a temperatura (isoniazida:ácido fumárico 2:130 e isoniazida:ácido 4-aminosalicílico 1:1). ${ }^{31}$ Esta ressalva também está baseada no fato de que o $\mathrm{pK}_{\mathrm{a}}$ é um parâmetro 
<smiles>O=C(O)c1ccccc1</smiles>

Ácido benzóico<smiles>NC(=O)c1cccnc1</smiles>

Nicotinamida<smiles>NC(=O)c1ccncc1</smiles>

Isonicotinamida<smiles>Oc1ccc(O)cc1</smiles>

Hidroquinona<smiles>O=C(O)c1ccc(O)cc1</smiles>

Ácido p-hidroxibenzóico<smiles>Nc1ccc(C(=O)O)cc1</smiles>

Ácido<smiles>Nc1ccccc1C(=O)O</smiles>

Ácido antranílico<smiles>O=C(O)c1ccccc1O</smiles>

Ácido salicílico<smiles>CC(=O)Oc1ccccc1C(=O)O</smiles>

Ácido acetilsalicílico<smiles>O=C(O)C(=O)O</smiles>

Ácido oxálico p-aminobenzóico<smiles>O=C(O)/C=C\C(=O)O</smiles>

Ácido maleico<smiles>O=C(O)CC(=O)O</smiles>

Ácido malônico<smiles>O=C(O)CCCC(=O)O</smiles>

Ácido glutárico<smiles>O=C(O)CC(O)(CC(=O)O)C(=O)O</smiles>

Ácido cítrico<smiles>O=C(O)CCC(=O)O</smiles>

Ácido succínico<smiles>O=C(O)C=CC(=O)O</smiles>

Ácido fumárico

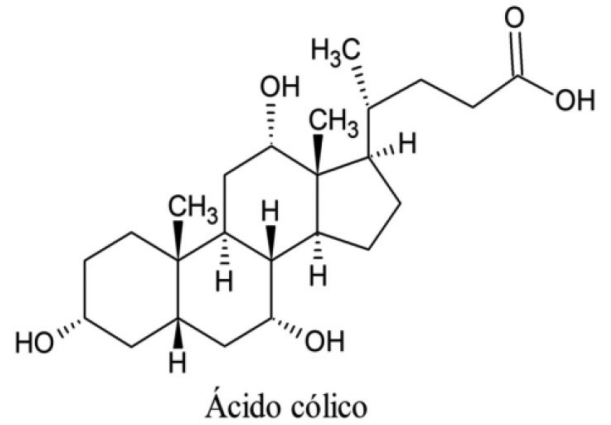<smiles>O=C1NS(=O)(=O)c2ccccc21</smiles><smiles>Cn1c(=O)c2c(ncn2C)n(C)c1=O</smiles>

Cafeína<smiles>Cn1c(=O)c2[nH]cnc2n(C)c1=O</smiles>

Teofilina

Figura 5. Relação de alguns dos coformadores mais utilizados na síntese de cocristais farmacêuticos. ${ }^{23,24}$

a<smiles></smiles>

b<smiles>CC(=O)NC1COCCO1</smiles>

$\mathrm{c}$<smiles></smiles>

Figura 6. Representação de synthons supramoleculares via ligação de hidrogênio entre ácidos e amidas: homosynthon entre dímeros ácido-ácido (a) e amida-amida (b); heterosynthon entre dímeros ácido-amida (c). Além dos synthons estabelecidos entre ácido-amida serem indiscutivelmente relevantes, synthons ácido-piridina também merecem destaque em cocristais farmacêuticos 
essencialmente definido em solução e pode não ser integralmente aplicável à predição de interações no estado sólido.

As dificuldades encontradas no desenho de estruturas cristalinas a partir de estruturas moleculares têm sido analisadas à luz da competição entre reconhecimento molecular (via synthons supramoleculares) e a estabilidade geométrica (empacotamento). É notável que quando as espécies químicas de um sistema estão relacionadas por ligações de hidrogênio fortes, o desenho de um cocristal se apresenta mais favorável e com resultados satisfatórios. No caso da predominância de interações moleculares fracas e da ocorrência de polimorfismo, a engenharia de cristais apresenta-se limitada. ${ }^{20,21}$ Estes obstáculos são um desafio para a predição de estruturas cristalinas a partir de modelos computacionais, sendo esta uma área ainda em desenvolvimento. ${ }^{32}$

Neste sentido, uma melhor compreensão do processo de cristalização (nucleação e química de superfície) vem sendo discutida como imprescindível para o desenvolvimento de estratégias eficazes para o desenho de materiais cristalinos em geral, inclusive cocristais. ${ }^{21,33,34}$ Além disso, trabalhos de triagem demonstram-se úteis na análise probabilística da ocorrência de synthons supramoleculares em materiais cocristalinos. A literatura está repleta de exemplos de engenharia de cristais aplicada à cocristalização e o crescente número de estudos realizados contribui para o desenvolvimento da área e para a superação dos desafios observados no presente momento.

\section{Exemplos de cocristais farmacêuticos}

Diversos estudos têm sido realizados aplicando a estratégia de cocristalização para fármacos. Na Tabela 1 encontram-se alguns exemplos de cocristais relatados na literatura que demonstraram aprimoramento das propriedades físico-químicas dos fármacos. A plataforma $\operatorname{CSD}^{22}$ foi utilizada na pesquisa (ConQuest 1.16$)^{35} \mathrm{e}$ visualização (Mercury 3.3) ) $^{36}$ de estruturas cristalinas.

\section{ESTRATÉGIAS DE FORMAÇÃO DE COCRISTAIS}

Diversas abordagens são utilizadas nos processos de cocristalização na área farmacêutica, dentre elas destacam-se a cristalização em solução, ${ }^{67-69}$ a moagem, ${ }^{70}$ a cristalização por fusão ${ }^{51,71}$ e o método de cristalização por reação (MCR). ${ }^{68}$

Entre os diferentes métodos, o $\mathrm{MCR}^{68}$ apresenta grande vantagem na triagem e obtenção de cocristais, uma vez que é capaz de formar cocristais sem que ocorra a cristalização dos componentes individuais. Este método baseia-se na geração de supersaturação da solução em relação ao complexo molecular a ser formado, no caso o cocristal, enquanto a solução encontra-se apenas saturada ou não saturada em relação aos componentes do cocristal. O diagrama de solubilidade de fases do cocristal (Figura 7), no qual este método é baseado, demonstra as condições não saturadas, saturadas e supersaturadas com relação ao cocristal e/ou às fases cristalinas individuais. Enquanto a cristalização ocorre em condições supersaturadas, em condições não saturadas ocorrerá a dissolução. Conforme ilustrado na Figura 7, o domínio I é supersaturado em relação ao componente A (fármaco), mas não saturado com respeito ao cocristal (AB). Ambos, $A$ e AB, estão supersaturados no domínio II, mas não saturados no domínio III. Por fim, o domínio IV é supersaturado com relação à $\mathrm{AB}$ mas não saturado com respeito a A. ${ }^{4,68,72}$ Portanto, a supersaturação relacionada unicamente ao cocristal pode ser gerada preparando-se soluções dos reagentes A e B de tal modo que as concentrações se encontrem na região IV. Esta condição pode ser alcançada através da mistura de soluções onde os reagentes estão dissolvidos, ou dissolvendo-se $\mathrm{o}$ (s) reagente(s) sólido(s) A e/ou B no solvente puro ou em soluções contendo os componentes sólidos. As fases sólidas A e B podem então transformar-se em cocristal $(\mathrm{AB})$ através de reação mediada em solução. Enquanto o cocristal AB é formado, ele consome A e B da solução e mais reagentes dissolvem-se até que o processo atinja o estado de equilíbrio determinado pela curva de solubilidade do cocristal. . $8,72^{2}$

A formação de cocristais pela adsorção de umidade ${ }^{73}$ ou solvente $^{74,75}$ de misturas físicas dos componentes do cocristal também é descrita pelo mesmo mecanismo do MCR.

Outros processos de cristalização em solução têm sido utilizados, onde razões estequiométricas dos componentes são dissolvidos em um solvente e, subsequentemente, evaporado ou aquecido, para facilitar a dissolução e, em seguida, resfriado (solvo-térmico). Estes métodos com base empírica apresentam um risco inerente de cristalizar uma ou mais fases indesejáveis incluindo componentes puros, além de exigirem, na maioria dos casos, a triagem de muitos solventes e/ou condições experimentais. Devido à variabilidade de evaporação ou mudanças de temperatura, pode haver dificuldades ao tentar aumentar a escala do processo de cristalização através destes métodos.

Métodos de cristalização em estado sólido via ativação mecânica, através da moagem dos componentes do cocristal, também são comumente empregados na área farmacêutica. ${ }^{39,69,76-82}$ Em alguns casos, fármaco e coformador apresentam uma fase intermediária amorfa. ${ }^{83}$ Desta forma, a temperatura de transição vítrea $\left(\mathrm{T}_{\mathrm{g}}\right)$ e a temperatura de fusão dos componentes são propriedades importantes para qualquer método mecanoquímico que induz transformação de fase e, portanto, devem ser consideradas. A moagem assistida por solvente emprega pequenos volumes de solvente durante a moagem e pode levar à formação do cocristal por processos mediados por: (1) alteração da mobilidade molecular da fase sólida e/ou (2) interações em solução. ${ }^{81,82}$

Além dos métodos mencionados, outro método que têm sido aplicado com sucesso na triagem e processos de obtenção de cocristais em grande escala é o método de fusão. ${ }^{5,71}$ A aplicação de fusão seguida de extrusão parece ser uma alternativa promissora para a formação de cocristais onde a instabilidade química não é um problema. ${ }^{84}$

Para confirmação da formação de um cocristal e diferenciação em relação a outros cristais multicomponentes, diversas técnicas de caracterização no estado sólido podem ser empregadas, como difração de raios X (DRX), ${ }^{85,86}$ análise térmica (Calorimetria Exploratória Diferencial - DSC e Termogravimetria -TGA), ${ }^{1,86,87}$ microscopia, ${ }^{88,89}$ espectroscopia vibracional (Raman e Infravermelho), ${ }^{86,90}$ espectroscopia do estado sólido (Ressonância magnética nuclear do estado sólido - RMNss) ${ }^{86,91-93}$ e análise de sorção de umidade. ${ }^{94-97}$

\section{PROPRIEDADES FÍSICO-QUÍMICAS DOS COCRISTAIS}

\section{Ponto eutético e constante eutética $\left(\mathbf{K}_{\mathrm{eu}}\right)$}

O ponto eutético é caracterizado pelas concentrações de fármaco e coformador ([fármaco $]_{\mathrm{eu}}$ e [coformador $]_{\mathrm{eu}}$ ) no ponto onde a solução encontra-se duplamente saturada em relação ao fármaco e cocristal. Neste ponto, a solubilidade do fármaco é igual à do cocristal e, a [fármaco $_{\mathrm{eu}}=[\text { coformador }]_{\mathrm{eu}}$, considerando um cocristal de razão estequiométrica $1: 1{ }^{4,9} \mathrm{O}$ ponto eutético é independente da massa de cada fase em equilíbrio, porém é dependente da temperatura, $\mathrm{pH}$, solvente e presença de agentes solubilizantes. A constante eutética $\left(\mathrm{K}_{\mathrm{eu}}\right)$ é uma importante indicadora da estabilidade do cocristal e é definida pela razão das atividades (a) do coformador e do fármaco no ponto eutético, as quais podem ser aproximadas à razão das concentrações (equação 1): ${ }^{98}$

$$
\mathrm{K}_{\mathrm{eu}} \equiv \frac{\mathrm{a}_{\text {coformador,eu }}}{\mathrm{a}_{\text {fármaco, eu }}} \approx \frac{\text { [coformador }_{\mathrm{eu}}}{[\text { fármaco }]_{\mathrm{eu}}}
$$


Tabela 1. Exemplos de cocristais farmacêuticos e propriedades do fármaco que foram aprimoradas

\begin{tabular}{|c|c|c|c|c|}
\hline Fármaco & $\begin{array}{l}\text { Classificação } \\
\text { SCB* }\end{array}$ & Coformador & Propriedades aprimoradas & Ref \\
\hline Aciclovir & IV & Ácido fumárico, ácido glutárico & $\begin{array}{l}\text { Solubilidade } \\
\text { Permeabilidade } \\
\end{array}$ & 37 \\
\hline AMG-517 & II & $\begin{array}{l}\text { Ácido glutárico, ácido glicólico, ácido sórbico, ácido trans-2- } \\
\text { hexanóico, ácido láctico, ácido benzóico }\end{array}$ & $\begin{array}{c}\text { Solubilidade } \\
\text { Taxa de dissolução }\end{array}$ & 38 \\
\hline Cafeína & I & Ácido oxálico & Estabilidade física & 39 \\
\hline Carbamazepina & II & Nicotinamida, sacarina, ácido 4-aminobenzóico & $\begin{array}{l}\text { Estabilidade física, } \\
\text { Taxa de dissolução e } \\
\text { Biodisponibilidade oral }\end{array}$ & 7,40 \\
\hline Cetoconazol & II & $\begin{array}{l}\text { Ácido fumárico, ácido succínico, ácido adípico, ácido } \\
\text { 4-aminobenzóico }\end{array}$ & $\begin{array}{l}\text { Taxa de dissolução } \\
\text { Solubilidade }\end{array}$ & 41,42 \\
\hline Etenzamida & II & $\begin{array}{l}\text { Ácido salicílico, ácido 2-cloro-4-nitrobenzóico, ácido } \\
\text { fumárico, ácido vanílico, ácido 4-aminobenzóico } \\
\text { ácido 4-hidroxibenzóico } \\
\end{array}$ & $\begin{array}{c}\text { Solubilidade } \\
\text { Velocidade de dissolução intrínseca }\end{array}$ & 43 \\
\hline Fluoxetina, cloridrato & $\mathrm{I}$ & Ácido benzoico, ácido succínico, ácido fumárico & Velocidade de dissolução intrínseca & 44 \\
\hline Furosemida & IV & $\begin{array}{l}\text { Acetamida, adenina, ácido 4-aminobenzóico, cafeína, citosina, } \\
\text { isonicotinamida, nicotinamida }\end{array}$ & $\begin{array}{c}\text { Estabilidade física } \\
\text { Solubilidade } \\
\text { Velocidade de dissolução intrínseca } \\
\end{array}$ & $45-47$ \\
\hline Gabapentina & III & Ácido 3-hidroxibenzóico & $\begin{array}{c}\text { Estabilidade física } \\
\text { Solubilidade } \\
\end{array}$ & 12 \\
\hline Griseofulvina & II & Acessulfamo & Solubilidade e taxa de dissolução & 48 \\
\hline Hidroclorotiazida & IV & $\begin{array}{l}\text { Ácido nicotínico, nicotinamida, ácido } p \text {-aminobenzóico, } \\
\text { succinimida }\end{array}$ & Solubilidade & 49 \\
\hline Ibuprofeno & II & 4,4'-dipiridilnicotinamida & Solubilidade & $50-52$ \\
\hline Indometacina & II & Sacarina & $\begin{array}{l}\text { Estabilidade física e } \\
\text { Taxa de dissolução }\end{array}$ & 19,53 \\
\hline Isoniazida & III & Nicotinamida, ácido succínico & $\begin{array}{c}\text { Solubilidade } \\
\text { Velocidade de dissolução intrínseca } \\
\end{array}$ & 54 \\
\hline Itraconazol & II & $\begin{array}{l}\text { Ácido fumárico, ácido L-málico, ácido succínico, ácido } \\
\text { D-tartárico, ácido DL-tartárico, ácido L-tartárico }\end{array}$ & Dissolução & 6 \\
\hline Meloxicam & II & Ácido acetilsalicílico & Farmacocinética & 55 \\
\hline Nevirapina & II & $\begin{array}{l}\text { Sacarina, ácido maleico, ácido glutárico, ácido salicílico, ácido } \\
\text { tartárico }\end{array}$ & $\begin{array}{c}\text { Solubilidade } \\
\text { Taxa de dissolução }\end{array}$ & 56 \\
\hline Nitrofurantoína & IV & Ácido 4-aminobenzóico, uréia & $\begin{array}{c}\text { Estabilidade física } \\
\text { Solubilidade } \\
\text { Velocidade de dissolução intrínseca }\end{array}$ & 57 \\
\hline Norfloxacino & IV & $\begin{array}{c}\text { Isonicotinamida, ácido succínico, ácido maleico, } \\
\text { ácido malônico }\end{array}$ & Solubilidade & 58 \\
\hline Pirazinamida & III & Ácido malônico, ácido glutárico, ácido succínico & $\begin{array}{c}\text { Solubilidade } \\
\text { Taxa de dissolução }\end{array}$ & 59 \\
\hline Piroxicam & II & $\begin{array}{l}\text { Ácido oxálico, ácido succínico, ácido salicílico, ácido } \\
\text { malônico, ácido fumárico }\end{array}$ & Solubilidade & 60 \\
\hline Quercetina & IV & Cafeína, isonicotinamida, teobromina & $\begin{array}{c}\text { Solubilidade } \\
\text { Biodisponibilidade }\end{array}$ & 61 \\
\hline Quinoxalina & & 3-tiosemicarbano-butan-2-ona-oxima (TSBO) & Solubilidade & 62 \\
\hline Sidenafila & $\mathrm{I}$ & Ácido acetilsalicílico & Velocidade de dissolução intrínseca & 63 \\
\hline Telmisartana & II & Sacarina, ácido glutárico & $\begin{array}{c}\text { Solubilidade } \\
\text { Biodisponibilidade } \\
\end{array}$ & 64 \\
\hline Temozolamida & I & Ácido oxálico, ácido salicílico, ácido succínico & Estabilidade química & 65 \\
\hline
\end{tabular}

* SCB = Sistema de Classificação Biofarmacêutica (leva em conta a solubilidade e a permeabilidade para classificar os fármacos em 4 classes: I - alta solubilidade e alta permeabilidade; II - baixa solubilidade e alta permeabilidade; III - alta solubilidade e baixa permeabilidade; IV - baixa solubilidade e baixa permeabilidade). ${ }^{66}$

Valores de $\mathrm{K}_{\mathrm{eu}}>1$ ou $\mathrm{K}_{\mathrm{eu}}>0,5$, para cocristais $1: 1$ e 2:1, respectivamente, indicam instabilidade termodinâmica do cocristal, porém maior solubilidade em relação ao fármaco quando em condições estequiométricas. Valores de $\mathrm{K}_{\mathrm{eu}}$ abaixo da razão estequiométrica, < 1 ou $<0,5$ para cocristais $1: 1$ e $2: 1$, respectivamente, indicam maior estabilidade termodinâmica (menor solubilidade) do cocristal em relação ao fármaco..$^{98}$

Cabe ressaltar que existem pelo menos dois pontos eutéticos, os quais são diferenciados pelas fases em equilíbrio: fármaco e cocristal ou coformador e cocristal. O ponto eutético mencionado acima refere-se ao fármaco e cocristal.

Ainda, as concentrações de fármaco e coformador no ponto eutético são utilizadas para acessar a solubilidade em equilíbrio do cocristal em soluções com diferentes condições, como valores de $\mathrm{pH}$ e presença de agentes solubilizantes. Os métodos experimentais de medida do ponto eutético encontram-se bem descritos 


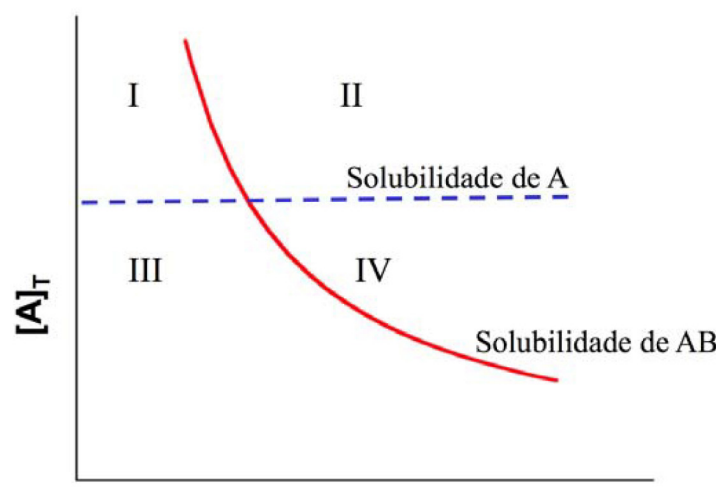

$[\mathrm{B}]_{\mathrm{T}}$

Figura 7. Solubilidade de um cocristal $A B$ em função da concentração de coformador $[B]_{T}$ Assume-se que solubilidade do fármaco A é independente da concentração de coformador. $[A]_{T}$ refere-se à concentração total do fármaco. Região IV: solução supersaturada em relação ao cocristal, o fármaco pode transformar-se em cocristal. Região I: solução supersaturada em relação ao fármaco, o cocristal pode converter à fármaco. Região II: solução supersaturada em relação ao fármaco e cocristal. Região III: solução não saturada. "Reproduzida com permissão de Nehm et al., 2006. ${ }^{72}$ Copyright 2006 American Chemical Society"

na literatura ${ }^{4,8,98,99}$ e requerem apenas: (1) pequenas quantidades de fármaco e cocristal em fase sólida suspensas na solução de interesse; (2) que a suspensão atinja saturação ou equilíbrio com relação às duas fases sólidas e à líquida; e (3) a medida das concentrações dos componentes do cocristal em solução. Devem ser medidos o pH e a temperatura, assim como faz-se necessário confirmar a presença das duas fases sólidas em equilíbrio. ${ }^{8}$

\section{$K_{\mathrm{sp}}$ e a solubilidade dos cocristais}

Uma propriedade importante dos cocristais é que sua solubilidade depende das atividades ou concentrações dos seus componentes em solução..$^{9,100}$ Durante a dissolução, os cocristais dissociam-se em seus constituintes e o equilíbrio entre a fase sólida e seus componentes em solução é descrito como um equilíbrio constante conhecido como o produto da solubilidade, $\mathrm{K}_{\mathrm{sp} .}{ }^{100}$ É importante lembrar que o termo "dissociação" se refere ao equilíbrio em solução, e não à precipitação dos componentes.

A solubilidade do cocristal diminui com o aumento da concentração de coformador conforme descrito pelo equilíbrio para um cocristal 1:1 RHA de um fármaco não ionizável (R) e um coformador ionizável (HA) (equação 2):

$$
\mathrm{RHA}_{\text {sólido }} \stackrel{\mathrm{K}_{\mathrm{sp}}}{\leftrightarrow} \mathrm{R}_{\text {aquoso }}+\mathrm{HA}_{\text {aquoso }}
$$

onde o produto da solubilidade, $\mathrm{K}_{\mathrm{sp}}$, é dado por:

$$
\mathrm{K}_{\mathrm{sp}}=[\mathrm{R}]_{\mathrm{aquoso}}[\mathrm{HA}]_{\mathrm{aquoso}}
$$

sendo [R] e [HA] as concentrações não ionizadas dos constituintes do cocristal.

A solubilidade estequiométrica do cocristal pode ser obtida a partir das concentrações eutéticas do fármaco e coformador ${ }^{99}$ utilizando a correlação descrita pela equação 4 :

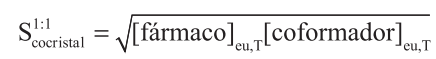

onde $\mathrm{T}$ subescrito é referente à concentração total em equilíbrio, e é dado pela soma de todas as espécies de fármaco e coformador em solução. Isto pode incluir as espécies ionizadas e não ionizadas, bem como as aquosas e solubilizadas.

Uma vez que obtida, a solubilidade do cocristal pode ser então utilizada para calcular o $\mathrm{K}_{\mathrm{sp}}$, de acordo com a equação 5:

$$
\mathrm{K}_{\mathrm{sp}}=\left(\mathrm{S}_{\text {cocristal, aquosa }}\right)^{2}
$$

É importante notar que o $\mathrm{K}_{\mathrm{sp}}$ é referente ao produto das concentrações aquosas livres e não ionizadas de fármaco e coformador.

A solubilidade do fármaco $\left(\mathrm{S}_{\mathrm{R}, \mathrm{T}}\right)$ é igual à concentração de fármaco no ponto eutético $[\mathrm{R}]_{\mathrm{eu}, \mathrm{T}}$, conforme a equação 6 :

$$
\mathrm{S}_{\mathrm{R}, \mathrm{T}}=[\mathrm{R}]_{\mathrm{eu}, \mathrm{T}}
$$

em condições de equilíbrio ( $\mathrm{pH}$, temperatura, concentração de coformador, concentração de agente solubilizante, etc.). Assim, tanto a solubilidade do cocristal como a do fármaco podem ser obtidas através do ponto eutético.

\section{Pontos de transição}

Apesar de ainda pouco difundidos na literatura, os pontos de transição são fundamentais para estabelecer as regiões de solubilidade e estabilidade termodinâmica dos cocristais. Um dado cocristal pode exibir maior, igual, ou menor solubilidade do que o fármaco dependendo da composição da solução, tais como concentração de coformador, $\mathrm{pH}$ e a presença de agentes solubilizantes do fármaco (Figura 8).4,8,10,14,15,99 Como resultado deste fenômeno, os cocristais exibem pontos de transição nos quais as solubilidades do fármaco e cocristal são iguais e, acima ou abaixo deste ponto, a vantagem da solubilidade do cocristal em relação ao fármaco é eliminada. Estes pontos de transição são caracterizados por: ${ }^{8,9}$

(1) $\mathrm{pH}_{\text {max }}$ : originado pela ionização dos componentes do cocristal,

(2) $S^{*}$ e CEC: gerados na presença de agentes solubilizantes devido à solubilização preferencial do fármaco em relação ao coformador, e

(3) $\mathrm{K}_{\mathrm{eu}}$ : originado por meio da dissolução do coformador em condições não-estequiométricas. Cada cocristal tem o seu $\mathrm{K}_{\mathrm{eu}}$ característico em solvente ou solução tampão e o seu valor é influenciado pelo $\mathrm{pH}$ e pela concentração de agentes solubilizantes.

$p H_{\max }$

O ponto de transição em função do pH ilustrado na Figura 8(a) demonstra um cocristal hipotético composto por um fármaco básico e um coformador ácido. A intersecção entre as duas curvas de solubilidade representa o ponto de transição deste sistema, o $\mathrm{pH}_{\max }$, que é o valor de $\mathrm{pH}$ onde a solubilidade do fármaco e do cocristal são iguais. ${ }^{10,12,101} \mathrm{O} \mathrm{pH}_{\max }$ é dependente do $\mathrm{K}_{\mathrm{sp}}$, da solubilidade aquosa do fármaco e dos valores de $\mathrm{pK}_{\mathrm{a}}$ dos componentes do cocristal. $\mathrm{O}$ cocristal é mais solúvel do que o fármaco em $\mathrm{pH}>\mathrm{pH}_{\max }$, similar ao caso de um cocristal com fármaco neutro e coformador ácido. ${ }^{10,12,101}$

Diversos cocristais relatados na literatura apresentam $\mathrm{pH}_{\max }$, por exemplo, cocristais dos fármacos itraconazol, gabapentina, piroxicam, lamotrigina, entre outros..$^{10,12,101,102} \mathrm{O} \mathrm{pH}_{\max }$ é também um parâmetro importante e bem descrito na literatura que identifica regiões de estabilidade de sais farmacêuticos. ${ }^{103-105}$

$S^{*} e C E C$

Agentes que solubilizam o fármaco tais como polímeros, surfactantes, lipídeos, entre outros, podem induzir pontos de transição. ${ }^{14-16,99,106,107}$ Dados recentes da literatura demonstram que este ponto de transição é caracterizado por um valor de solubilidade e de concentração do agente solubilizante. ${ }^{16} \mathrm{O}$ ponto em que a 


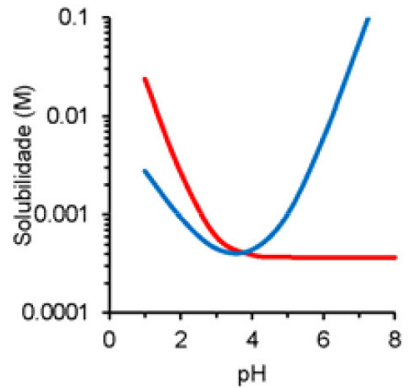

(a)

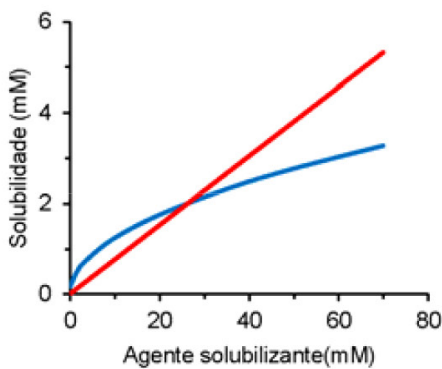

(b)

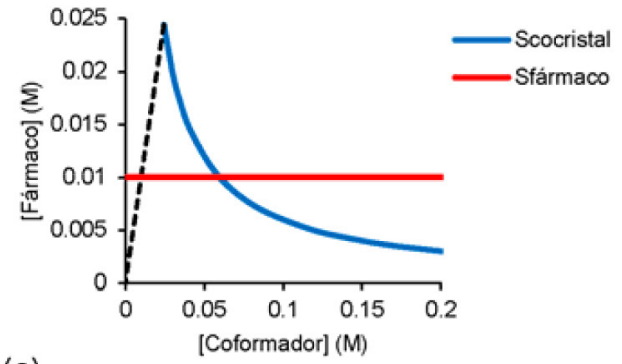

(c)

Figura 8. Influência do (a) pH, (b) agente solubilizante do fármaco, e (c) concentração de coformador na solubilidade e estabilidade termodinâmica do cocristal. A composição da solução modifica a solubilidade do cocristal em relação à do fármaco e da mesma forma, a estabilidade termodinâmica do cocristal. $O$ cocristal é termodinamicamente estável quando $S_{\text {cocristal }} \leq S_{\text {fármaco. }}$ O ponto de transição é a interseção entre a $S_{\text {cocristal }}$ e a $S_{\text {fármaco }}$ Adaptada de Kuminek et al. (2016), ${ }^{8}$ com permissão da Elsevier

solubilidade do fármaco e do cocristal são iguais na presença de um agente solubilizante é definido como $\mathrm{S}^{*}$, e a concentração de agente solubilizante neste ponto é chamada de concentração de estabilização crítica (CEC). Nos casos em que o coformador não interage com o agente solubilizante, o $\mathrm{S}^{*}$ pode ser determinado unicamente pela solubilidade aquosa do fármaco e do cocristal. ${ }^{16} \mathrm{O}$ CEC obedece o mecanismo de solubilização preferencial, variando de acordo com a natureza do agente solubilizante..$^{15,99}$ Ambos S* e CEC irão variar de acordo com a solubilidade aquosa do cocristal. Cocristais com maior solubilidade aquosa apresentarão maior valor de $\mathrm{S}^{*}$ e CEC. ${ }^{16}$

$\mathrm{O}$ valor de $\mathrm{S}^{*}$ é independente do agente solubilizante, com a condição de que o coformador não seja solubilizado pelo mesmo, como ilustrado na Figura 9. Entretanto, $\mathrm{S}^{*}$ é dependente de fatores que alteram a solubilidade aquosa do fármaco e do cocristal, tais como pH e ionização dos componentes em solução. ${ }^{16}$

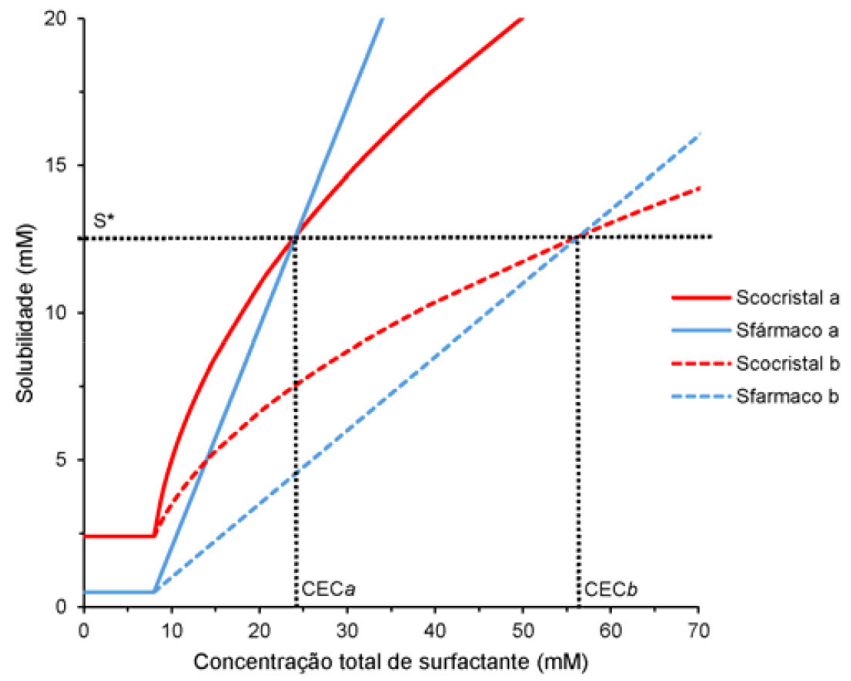

Figura 9. Pontos de transição $S^{*}$ e CEC para o mesmo cocristal em meios contendo dois agentes solubilizantes diferentes, a e b. O valor de $S^{*}$ é constante e o CEC varia de acordo com a extensão da solubilização do fármaco pelo agente solubilizante. $O$ fármaco apresenta maior solubilização pelo agente a do que pelo b, logo CECa $<C E C b$. Reproduzida com permissão de Lipert e Rodríguez-Hornedo, 2015. ${ }^{16}$ Copyright 2015 American Chemical Society

Cocristais de CBZ, DNZ, IND e PTB apresentam S* e CEC em soluções contendo agentes solubilizantes do fármaco. ${ }^{14-16,99,107,108}$ Como indicado na Figura 10(a), o cocristal PTB-CAF está acima dos valores de $\mathrm{S}^{*}$ e CEC enquanto DNZ-HBA em $150 \mathrm{mM}$ de Tween 80 encontra-se abaixo. Na Figura 10(c), para o cocristal CBZ-SAC, a intersecção das curvas de solubilidade do fármaco e cocristal é caracterizada por um valor de solubilidade ( $\mathrm{S}^{*}$ ) e uma concentração de LSS (CEC) ${ }^{15,16}$ Os valores de $\mathrm{S}^{*}$ e CEC também podem ser obtidos pelo gráfico de solubilidade $v s$ concentração de agente solubilizante, como mencionado anteriormente. Ainda, podem ser utilizadas relações matemáticas para prever $\mathrm{S} *$ e CEC pelo conhecimento da vantagem da solubilidade (na presença ou ausência de agentes solubilizantes) e a razão de solubilização do fármaco $\left(\mathrm{RS}_{\text {fármaco }}\right)$ e, desta forma, apenas um número limitado de experimentos é requerido. ${ }^{8}$ A Figura 10(b) apresenta os valores preditos e experimentais de SA para os cocristais de CBZ, DNZ e PTB em função da $\mathrm{RS}_{\text {fármaco }}$ em diferentes sistemas de surfactantes. Os cocristais são mais solúveis do que o fármaco na faixa de $\mathrm{RS}_{\text {fármaco }}$ onde $\mathrm{SA}>1$, e menos solúveis do que o fármaco na faixa de $\mathrm{RS}_{\text {fármaco }}$ onde $\mathrm{SA}<1$. Estes pontos de transição podem ser determinados experimentalmente por diferentes abordagens que dependem das medidas de solubilidade na presença ou na ausência de agentes solubilizantes.

O conhecimento sobre os pontos de transição permite modular o aumento de solubilidade do cocristal em relação ao fármaco através de um simples ajuste do $\mathrm{pH}$ e/ou da concentração do agente solubilizante. Assim, a supersaturação gerada durante a dissolução do cocristal pode ser modulada e, desta forma, controlar a conversão de fases de maneira racional.

\section{SOLUBILIDADE DE COCRISTAIS}

A versatilidade dos cocristais no aprimoramento da solubilidade de fármacos faz desta propriedade uma das suas características físico-químicas mais investigadas, uma vez que, para ser absorvido o fármaco necessita ser primeiramente dissolvido. ${ }^{1}$ Aprimorar a solubilidade de um fármaco pode levar ao aumento da sua taxa de dissolução e, consequentemente, da sua biodisponibilidade. ${ }^{66}$

A solubilidade de um cocristal é determinada por dois fatores independentes: a força da rede cristalina e a solvatação dos componentes do cocristal. Assim, o aumento da solubilidade pode estar relacionado com a diminuição da energia da rede cristalina do cocristal e/ou com uma maior afinidade pelo solvente, oriunda de diferentes características superficiais. ${ }^{100}$ Desta forma, as estratégias utilizadas no aprimoramento da solubilidade envolvem uma combinação de tecnologias que podem influenciar as forças de interação entre solvente-solvente, solvente-soluto e soluto-soluto. As forças de interação soluto-soluto devem ser superadas para que o soluto possa se dissolver. No caso de soluções ideais, quando as interações solvente-soluto somam-se às interações solvente-solvente, a energia de solubilização é dada pela energia de rede. ${ }^{9}$ A Figura 11 ilustra os passos envolvidos no processo de solubilização ou dissolução. 
(a)

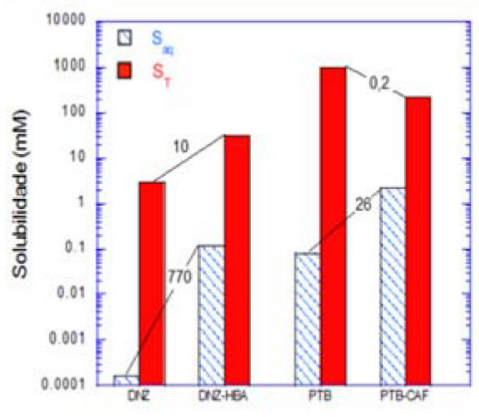

(b)

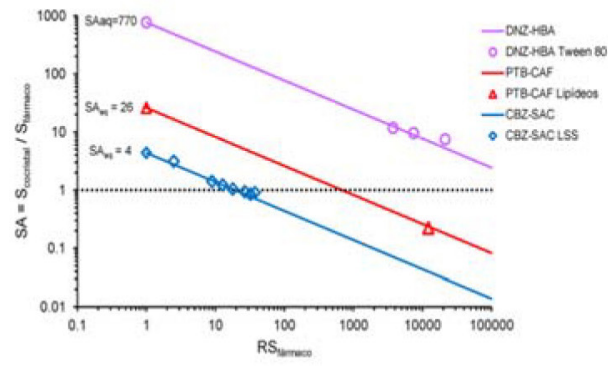

(c)

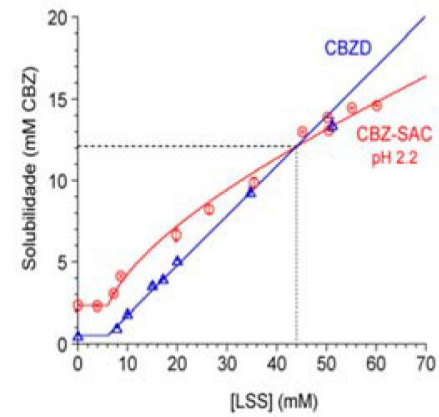

Figura 10. A vantagem da solubilidade do cocristal sobre o fármaco diminui quando o fármaco é preferencialmente solubilizado por aditivos, tais como surfactantes e lipídeos. (a) as solubilidades dos cocristais de DNZ e PTB são muito maiores do que a solubilidade do fármaco em solução aquosa, mas esta vantagem é diminuída e pode até ser invertida em soluções contendo aditivos. (b) a diminuição na SA e no valor de $S^{*}$ pode ser predita a partir do conhecimento da razão de solubilização do fármaco $\left(R S_{\text {fármaco }}\right)$ de acordo com $S A=\frac{S A_{\text {aquoso }}}{\sqrt{R S_{\text {farmaco }}}}$. O cocristal está no ponto de transição quando o $S A=1$; abaixo do ponto de transição quando $S A>1$; e acima quando $S A<1$. O CEC e $S^{*}$ podem ser obtidos a partir da concentração do agente solubilizante e da solubilidade do fármaco, respectivamente, pelo valor de $R S_{\text {fármaco }} q u e$ corresponde a $S A=1$. Os símbolos representam medidas experimentais e as linhas foram obtidas a partir da equação para $S A$ descrita acima. A linha pontilhada representa os valores de $R S_{\text {färmaco }}$ no ponto de transição. (c) Influência da concentração de surfactante na solubilidade do fármaco e cocristal indicando que a diferente dependência de cada um leva ao ponto de transição caracterizado por $S^{*}$ e CEC. Os símbolos representam valores experimentais e as linhas representam a solubilidade predita pelas equações $S_{\text {färmaco,T }}=S_{\text {färmaco,aq }}\left(1+K_{s}^{\text {färmaco }}[M]\right)$ para o fármaco (linha azul) e a equação 14 para o cocristal (linha vermelha). Adaptada de Cocrystals to facilitate delivery of poorly soluble compounds beyond-rule-of-5, 101, Kuminek, G.; Cao, F.; Rocha, A.B.O.; Cardoso, S. G.; Rodríguez-Hornedo, N., 143-166, Copyright 2016, com permissão da Elsevier ${ }^{8}$ - Adaptada de Journal of Pharmaceutical Sciences, 100, Huang, N.; Rodríguez-Hornedo, N. Engineering Cocrystal Solubility, Stability, and pHmax by Micellar Solubilization, 5219-5234, Copyright 2011, com permissão da Elsevier ${ }^{15}$

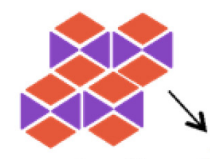

rede cocristalina (soluto-soluto)

(1)

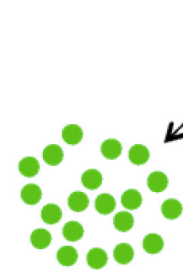$$
\text { (1) }
$$

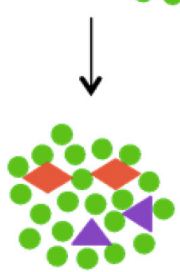

solução

(solvente-soluto)

(3)

Figura 11. Representação dos passos envolvidos no processo de solubilização de um cocristal. (1) quebra das ligações intermoleculares do sólido (soluto-soluto); (2) quebra das ligações intermoleculares do solvente (solvente-solvente); (3) formação de ligações solvente-soluto. Adaptado da ref ${ }^{9}$ com permissão da "The Royal Society of Chemistry"

Os cocristais podem gerar supersaturação em solução em relação à solubilidade do fármaco. No entanto, cocristais altamente solúveis também podem transformar-se rapidamente no fármaco (termodinamicamente mais estável e menos solúvel), tornando a tentativa de medir a solubilidade do cocristal por dissolução ou suspensão de cocristais potencialmente instável (Figura 12). ${ }^{9}$

À medida que ocorre a dissolução de um cocristal moderadamente solúvel, este pode atingir um máximo de concentração em solução e em seguida dar início à cristalização do fármaco estável (conversão cinética). No entanto, esta concentração que corresponde ao pico $\left(\mathrm{C}_{\max }\right)$ não deve ser considerada a solubilidade do cocristal.

Por outro lado, cocristais altamente solúveis, por apresentarem o potencial de gerar uma supersaturação extremamente alta em relação ao fármaco, quando submetidos a ensaios de dissolução ficam

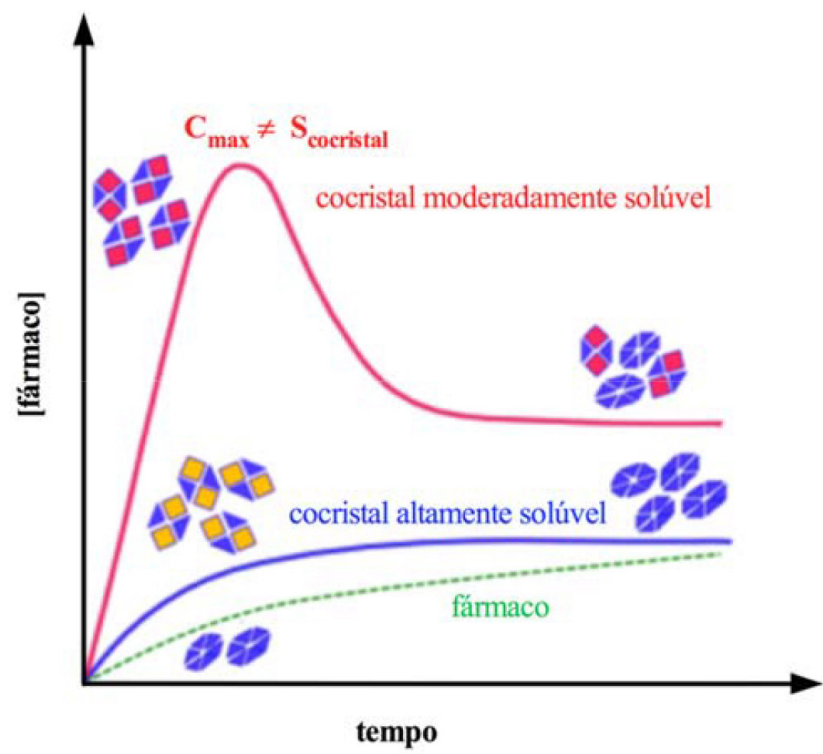

Figura 12. Concentração de fármaco ([fármaco]) em função do tempo para cocristais que apresentam diferentes solubilidades. À medida que o cocristal dissolve e o fármaco precipita, as concentrações de fármaco podem atingir um máximo no caso de cocristais com solubilidade moderada, enquanto que cocristais altamente solúveis podem precipitar imediatamente em fármaco e a concentração de fármaco é mantida perto ou na mesma solubilidade do fármaco. Adaptada de Kuminek et al. (2016), ${ }^{8}$ com permissão da Elsevier

susceptíveis à precipitação em fármaco de forma imediata. Assim, devido a esta rápida conversão, a concentração em solução é mantida próxima ou igual à da solubilidade do fármaco estável, resultando algumas vezes em valores subestimados ou até mesmo errôneos, que não devem ser relacionados à solubilidade termodinâmica.

Apesar das óbvias falhas de medir a solubilidade do cocristal desta forma, muitos estudos ainda utilizam o "plateau" ou " $C_{\max }$ " da 
dissolução do cocristal como a sua solubilidade, não percebendo que a verdadeira solubilidade do cocristal foi desperdiçada.

A solubilidade de um cocristal pode ser investigada através de métodos termodinâmicos e cinéticos. Medidas cinéticas são geralmente o primeiro e mais comum método utilizado. ${ }^{41,100,109,110}$ No entanto, esse método normalmente não consegue captar o verdadeiro perfil de concentração do cocristal, em vez disso, um perfil de concentração do fármaco é revelado como resultado da conversão cinética do cocristal. Como consequência, o poder dos cocristais de aumentar a solubilidade pode ser subestimado através do uso de medições cinéticas. ${ }^{9}$ As informações obtidas através das medições em equilíbrio (termodinâmicas) proporcionam conhecimento sobre o comportamento do cocristal em solução, que é transferível para outras condições. Através de estudos termodinâmicos é possível delinear os estudos cinéticos (por exemplo, seleção de aditivos e condições de $\mathrm{pH}$ ). Conhecer a pureza do cocristal e as condições da solução é fundamental para obter-se a medida correta da solubilidade do cocristal. ${ }^{100}$ Tanto os conceitos termodinâmico quanto cinético são importantes para o desenvolvimento de sistemas que promovem a supersaturação de fármacos, como os cocristais.

\section{MODULAÇÃO DA SOLUBILIDADE DOS COCRISTAIS POR MEIO DE INTERAÇÕES EM SOLUÇÃO}

Como resultado de suas propriedades moleculares diferentes, e por apresentarem múltiplos componentes, as associações moleculares em solução contribuem de forma importante para a solubilidade dos cocristais, conforme mostra a Figura $13 .{ }^{9}$ À medida que o cocristal dissolve em solução, seus constituintes dissociam-se e podem interagir com a solução por diferentes processos moleculares, incluindo complexação, ionização, bem como a solubilização micelar em meio aquoso e biorrelevante. Essas interações, por sua vez, influenciarão a solubilidade de equilíbrio do cocristal. Não somente a solubilidade do cocristal irá variar com base nas condições em solução, mas também a sua vantagem de solubilidade em relação à solubilidade do fármaco. ${ }^{8}$

\section{Ionização}

Além de aumentar a solubilidade, os cocristais podem modular a solubilidade em função do $\mathrm{pH}$ devido às propriedades de ionização dos coformadores que são, por exemplo, moléculas ácidas, básicas, anfotéricas ou zwiteriônicas. A habilidade de prever esse comportamento é importante a fim de conhecer a solubilidade aquosa desejada e então caracterizar a solubilidade dos cocristais dependentes do $\mathrm{pH}$. Estudos com diversos cocristais que demonstram este comportamento encontram-se reportados na literatura. ${ }^{10-12,101}$

Para um cocristal com componentes ionizáveis, a solubilidade é governada pelo produto de solubilidade $\left(\mathrm{K}_{\mathrm{sp}}\right)$, constantes de ionização $\left(\mathrm{K}_{\mathrm{a}}\right)$, e o pH da solução. ${ }^{9,100} \mathrm{Na}$ maioria dos casos, os valores de $\mathrm{K}_{\mathrm{a}}$ são conhecidos e o $\mathrm{K}_{\mathrm{sp}}$ pode ser calculado a partir da solubilidade do cocristal experimentalmente medida em um determinado $\mathrm{pH}$. Alternativamente, pode-se atingir valores de solubilidade e $\mathrm{pH}$, e então calcular os valores de $\mathrm{K}_{\mathrm{a}}$ e $\mathrm{K}_{\mathrm{sp}}$. As equações que descrevem a dependência da solubilidade do cocristal em $\left[\mathrm{H}^{+}\right], \mathrm{K}_{\mathrm{sp}}$, e $\mathrm{K}_{\mathrm{a}}$ com componentes ácidos, básicos, anfotéricos e zwiteriônicos foram derivadas e estão bem documentadas em literatura. ${ }^{10}$ Estas equações aplicam-se a cocristais que estão em equilíbrio com as concentrações em solução de estequiometria diferente ou igual ao cocristal.

A solubilidade de um cocristal 1:1 RHA, onde R é um fármaco não ionizável e HA é um coformador ácido, pode ser determinada considerando o equilíbrio do cocristal em solução. Se este equilíbrio é descrito pela dissociação do cocristal e ionização do coformador, as

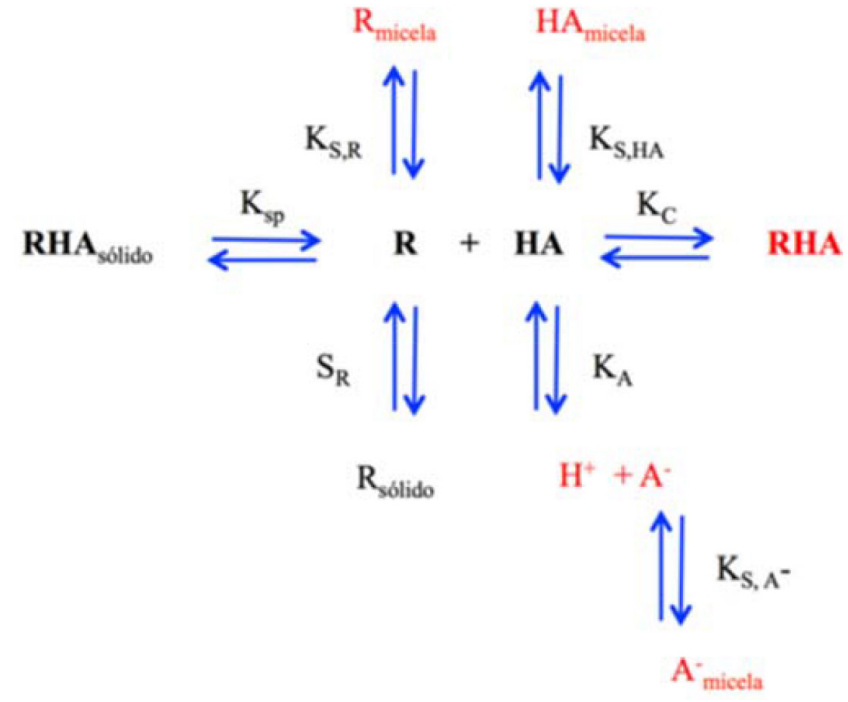

Figura 13. Interações entre as fases em solução e respectivo equilíbrio para um cocristal RHA de um fármaco não ionizável $(R)$ e um coformador ácido (HA) em solução micelar. Adaptado da ref ${ }^{9}$ com permissão da "The Royal Society of Chemistry"

reações de equilíbrio e suas constantes são dadas pelas equações 7 e 8 :

$$
\begin{gathered}
\mathrm{RHA}_{\text {sólido }} \stackrel{\mathrm{K}_{\mathrm{sp}}}{=} \mathrm{R}_{\mathrm{sol}}+\mathrm{HA}_{\text {sol }} \\
\mathrm{K}_{\mathrm{sp}}=[\mathrm{R}]_{\text {aquoso }}[\mathrm{HA}]_{\text {aquoso }} \\
\mathrm{HA}_{\text {sol }} \stackrel{\mathrm{K}_{\mathrm{a}}}{=} \mathrm{A}_{\mathrm{sol}}^{-}+\mathrm{H}_{\mathrm{sol}}^{+} \\
\mathrm{K}_{\mathrm{a}}=\frac{\left[\mathrm{H}^{+}\right]\left[\mathrm{A}^{-}\right]}{[\mathrm{HA}]}
\end{gathered}
$$

A análise aqui apresentada assume soluções diluídas, onde as atividades são relativamente constantes e podem ser substituídas por concentrações nas constantes de equilíbrio, sendo uma aproximação para estabelecer tendências gerais. Condições não ideais devido à complexação, interações iônicas e interações solvente-soluto deverão ser consideradas para uma análise mais rigorosa, particularmente em concentrações e forças iônicas elevadas. ${ }^{9}$

Quando um cocristal está em equilíbrio com soluções de uma estequiometria igual a sua, por exemplo, quando não há excesso de fármaco ou coformador em solução, sua solubilidade é igual à concentração total (soma das espécies ionizadas e não ionizadas) de fármaco ou coformador em solução (equação 9):

$$
\begin{gathered}
\mathrm{S}_{\text {cocristal }}=[\mathrm{R}]_{\mathrm{T}}=[\mathrm{A}]_{\mathrm{T}} \\
\mathrm{S}_{\text {cocristal }}=[\mathrm{R}]_{\text {aquoso }}=[\mathrm{HA}]_{\text {aquoso }}+\left[\mathrm{A}^{-}\right]_{\text {aquoso }}
\end{gathered}
$$

Desta forma, a equação 9 é substituída por:

$$
\mathrm{S}_{\text {cocristal }}=\sqrt{\mathrm{K}_{\mathrm{sp}}\left(1+\frac{\mathrm{K}_{\mathrm{a}}}{\left[\mathrm{H}^{+}\right]}\right)}
$$

Nessas condições, a solubilidade é reportada como solubilidade estequiométrica.

A equação 11 prediz que a solubilidade do cocristal RHA irá aumentar com a diminuição da $\left[\mathrm{H}^{+}\right]$, ou seja, com o aumento do $\mathrm{pH}$. 
Conforme mencionado anteriormente, a solubilidade do cocristal também é dependente do $\mathrm{K}_{\mathrm{sp}}$ do cocristal e da $\mathrm{K}_{\mathrm{a}}$ do coformador. Em um $\mathrm{pH} \ll \mathrm{pK}_{\mathrm{a}}$ do coformador, ou $\left[\mathrm{H}^{+}\right] \gg \mathrm{K}_{\mathrm{a}}$, a solubilidade do cocristal se aproxima da solubilidade intrínseca $\sqrt{K_{s p}}$. Em um $\mathrm{pH}=\mathrm{pK}_{\mathrm{a}}$, ou $\left[\mathrm{H}^{+}\right]=\mathrm{K}_{\mathrm{a}}$, a solubilidade do cocristal é $\sqrt{2 K_{s p}}$ ou 1,4 vezes a solubilidade intrínseca do cocristal. Em um $\mathrm{pH} \gg>\mathrm{pK}_{\mathrm{a}}$ do coformador, ou $\left[\mathrm{H}^{+}\right]<\mathrm{K}_{\mathrm{a}}$, a solubilidade do cocristal aumenta exponencialmente. No entanto, a concentração máxima que pode ser obtida experimentalmente é limitada pela solubilidade do fármaco e do coformador. ${ }^{9}$

Para um cocristal com um fármaco não ionizável e um coformador básico é prevista uma dependência inversa (equação 12):

$$
\mathrm{S}_{\text {cocristal }}=\sqrt{\mathrm{K}_{\mathrm{sp}}\left(1+\frac{\left[\mathrm{H}^{+}\right]}{\mathrm{K}_{\mathrm{a}}}\right)}
$$

onde $\mathrm{K}_{\mathrm{a}}$ é a constante de ionização do ácido conjugado da base. Neste caso, a solubilidade irá aumentar com o aumento da $\left[\mathrm{H}^{+}\right]$, ou seja, diminuição do $\mathrm{pH}$.

Através desta análise é possível intuir que cocristais conferem uma solubilidade $\mathrm{pH}$-dependente à fármacos não ionizáveis quando os coformadores são ionizáveis.

Conforme previamente demonstrado na Figura 4, cocristais de um fármaco não ionizável podem exibir um perfil de solubilidade pH-dependente completamente diferente, dependendo das propriedades de ionização do coformador. Um coformador ácido diprótico irá levar a um aumento da solubilidade com o aumento do $\mathrm{pH}$, assim como um ácido monoprótico. Um coformador anfotérico irá resultar em um perfil em formato de U com a solubilidade mínima em um $\mathrm{pH}$ entre os dois valores de $\mathrm{pK}_{\mathrm{a}}$. Perfil similar é previsto para um cocristal de um fármaco básico e um coformador ácido, onde os grupos ionizáveis estão presentes em diferentes moléculas. Este perfil também é esperado para um cocristal de um fármaco zwiteriônico e coformador ácido. ${ }^{10}$

Compreender a dependência da solubilidade do cocristal em função do $\mathrm{pH}$ e da concentração de coformador é importante para determinar os diagramas de fase e identificar as regiões de estabilidade termodinâmica. Esta análise mostra que a solubilidade do cocristal e as regiões de estabilidade podem ser estimadas através de uma única medição da solubilidade do cocristal e do $\mathrm{pH}$, não sendo necessário determinar experimentalmente todo o diagrama de fase. ${ }^{10}$

\section{Agentes solubilizantes}

Muitos agentes solubilizantes, tais como agentes tensoativos, polímeros e lipídios, são empregados na indústria farmacêutica para melhorar a solubilidade de fármacos pouco solúveis em meio aquoso. ${ }^{63-65,73-75} \mathrm{O}$ teor lipídico presente nos alimentos e a presença de sais biliares também podem melhorar o desempenho de um fármaco lipofílico/hidrofóbico in vivo. ${ }^{111-115}$ No entanto, o efeito destes agentes de solubilização na solubilidade do cocristal geralmente não é tão simples. Cocristais farmacêuticos são geralmente compostos por um fármaco hidrofóbico e um coformador relativamente hidrofílico. Portanto, espera-se que os constituintes do cocristal apresentem diferentes afinidades pelos agentes solubilizantes, mecanismo conhecido como solubilização preferencial. Dessa forma, dependendo da concentração e da natureza do agente solubilizante, um cocristal pode exibir solubilidade maior, igual, ou menor do que o fármaco constituinte.

A implicação prática dos fatores que determinam a solubilidade do cocristal em relação ao fármaco constituinte em meios contendo aditivos que solubilizam fármacos pouco solúveis em água necessita ser bem compreendida, uma vez que diversos agentes solubilizantes são frequentemente encontrados em formulações e nos meios de dissolução (in vivo e in vitro).

A relação entre a razão de solubilização do cocristal e do fármaco pode ser obtida através da associação das equações que descrevem a solubilidade do cocristal e do fármaco na presença de agentes solubilizantes. ${ }^{14,15,99}$ Para um cocristal 1:1 sob condições não ionizáveis, onde a sua solubilidade é dada pela equação 13 :

$$
\mathrm{S}_{\text {cocristal }, \mathrm{T}}=\sqrt{\mathrm{K}_{\mathrm{sp}}\left(1+\mathrm{K}_{\mathrm{s}}^{\text {fármaco }}[\mathrm{M}]\right)\left(1+\mathrm{K}_{\mathrm{s}}^{\text {coformador }}[\mathrm{M}]\right)}
$$

$\mathrm{S}_{\text {cocristal, } \mathrm{T}}$ representa a soma das solubilidades do cocristal em meio aquoso e micelar, $\mathrm{S}_{\text {cocristal, } \mathrm{T}}=\mathrm{S}_{\text {cocristal,aquoso }}+\mathrm{S}_{\text {cocristal,micelar }}$ Onde $\mathrm{K}_{\mathrm{sp}}$ é o produto da solubilidade, $\mathrm{K}_{\mathrm{sp}}=\left(\mathrm{S}_{\text {cocristal,aquoso }}\right)^{2}$. $\mathrm{K}_{\mathrm{s}}$ significa constante de solubilização dos constituintes cocristal. [M] representa a concentração micelar do agente solubilizante, calculada pela concentração total do agente solubilizante menos sua concentração micelar crítica (CMC).

Considerando a solubilização preferencial, em meio aquoso os agentes solubilizantes tendem a interagir preferencialmente com fármacos, uma vez que os fármacos que constituem o cocristal são geralmente hidrofóbicos, enquanto coformadores são hidrofílicos.

Quando o agente solubilizante aumenta a solubilidade somente do fármaco, e não do coformador $\left(\mathrm{K}_{\mathrm{s}}^{\text {coformador }}=0\right)$, a solubilidade do cocristal (equação 13) é substituída gerando a equação 14 :

$$
\mathrm{S}_{\text {cocristal }, \mathrm{T}}=\sqrt{\mathrm{K}_{\mathrm{sp}}\left(1+\mathrm{K}_{\mathrm{S}}^{\text {fármaco }}[\mathrm{M}]\right)}
$$

Este comportamento pode ser previsto, como mostrado na Figura 10. Agentes solubilizantes do fármaco influenciam particularmente a vantagem da solubilidade do cocristal em relação ao fármaco. Um cocristal mais solúvel irá apresentar uma maior vantagem da solubilidade na ausência de agente solubilizante, entretanto, a medida que a concentração de agente solubilizante aumenta, esta vantagem da solubilidade diminui. Desta forma, agentes solubilizantes podem induzir pontos de transição. ${ }^{14-16,27,106,107} \mathrm{Um}$ exemplo interessante é o cocristal de indometacina-sacarina (IND-SAC), cuja vantagem da solubilidade, 26 vezes maior do que a indometacina em $\mathrm{pH} 2,{ }^{11}$ é eliminada na presença de lauril sulfato de sódio, Brij 99 ou Tween 80 , onde o cocristal apresenta menor solubilidade. ${ }^{11,107}$ Os agentes solubilizantes podem, de fato, aumentar a solubilidade de ambos, cocristal e fármaco, porém a razão da solubilização $\left(\mathrm{S}_{\text {cocristal }} / \mathrm{S}_{\text {fármaco }}\right)$ é variável, dependendo da natureza e da concentração destes agentes.

Para um cocristal 1:1, a relação entre a razão de solubilização (RS) do cocristal e do fármaco, é definida pela equação 15 :

$$
\left(\frac{\mathrm{S}_{\mathrm{T}}}{\mathrm{S}_{\text {aquoso }}}\right)_{\text {cocristal }}=\sqrt{\left(\frac{\mathrm{S}_{\mathrm{T}}}{\mathrm{S}_{\text {aquoso }}}\right)}
$$

De forma simplificada, RS do cocristal 1:1 corresponde à raiz quadrada da RS do fármaco (equação 16):

$$
\mathrm{RS}_{\text {cocristal }}=\sqrt{\mathrm{RS}_{\text {färmaco }}}
$$

As equações apresentadas neste trabalho, encontram-se bem fundamentadas e descritas na literatura, assim como modelos matemáticos referentes a cocristais de diferentes estequiometrias e envolvendo fármacos ionizáveis. ${ }^{16,116}$

A Figura 14 demonstra a solubilização preferencial de agentes solubilizantes (sintéticos e biorrelevantes) por fármacos hidrofóbicos para cocristais de carbazepina (CBZ), indometacina (IND), danazol (DNZ), com coformadores hidrofílicos. ${ }^{14,15,99,106-108,117-119}$ 

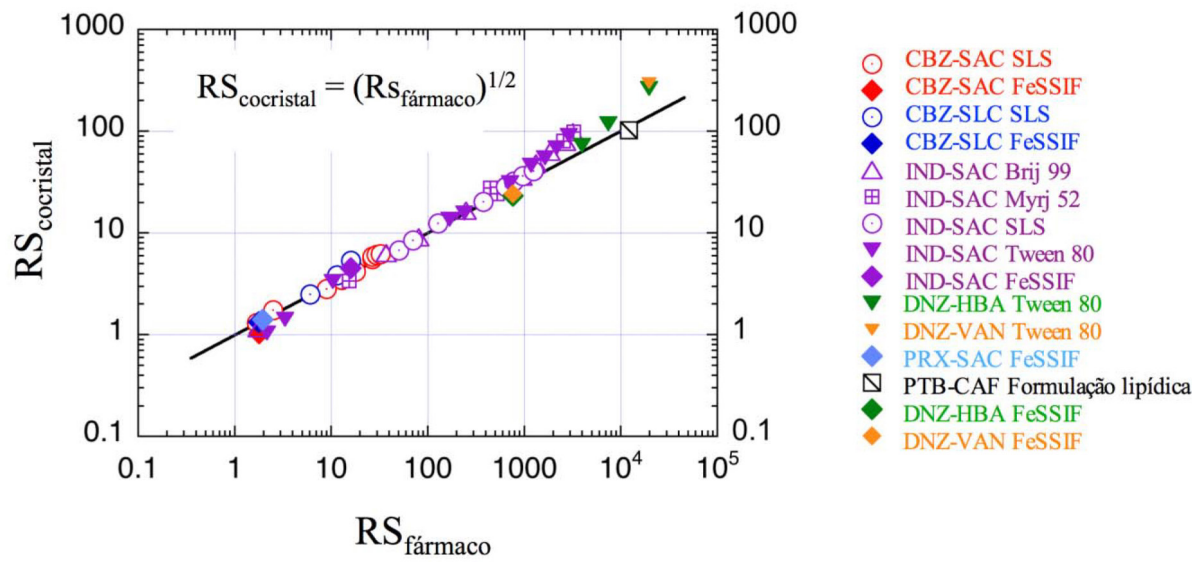

Figura 14. Dependência da razão da solubilidade do cocristal $\left(R S_{\text {cocristal }}\right)$ na razão de solubilidade do fármaco $\left(R S_{\text {färmaco }}\right)$ para cocristais 1:1. Linhas representam a relação teórica entre $R S_{\text {cocristal }}$ e $R S_{\text {fármaco }}$ de acordo com a equação (16). O declive predito da linha é 1/2. Símbolos representam valores de $R S$ determinados experimentalmente para cocristais e fármacos medidos em condições de equilíbrio com agentes solubilizantes. Carbamazepina (CBZ), sacarina (SAC), SLS (lauril sulfato de sódio), FeSSIF (fluido intestinal simulado alimentado), indometacina (IND), danazol (DNZ), ácido hidróxi-benzóico (HBA), vanilina (VAN), PRX (piroxicam), pterostilbeno (PTB), cafeína (CAF). Reproduzida com permissão de Lipert e Rodríguez-Hornedo, 2015. ${ }^{16}$ Copyright 2015 American Chemical Society

\section{CONSIDERAÇÕES FINAIS}

Estudos envolvendo sólidos cristalinos desempenham importantes papéis na área de fármacos, influenciando áreas da pesquisa como a biofarmácia, a tecnologia da produção, o desenvolvimento de formas farmacêuticas, a purificação de matérias primas, o controle de qualidade e seus estudos de estabilidade, a manufatura, os aspectos regulatórios e as patentes de medicamentos, e o marketing. Dentro da classe dos sistemas cristalinos, os cocristais surgem como uma estratégia promissora nas pesquisas de aprimoramento de moléculas pouco solúveis e têm gerado, nos últimos anos, uma série de conceitos, paradigmas e modelos de estudo, ganhando interesse tanto da área de engenharia de cristais como da farmacêutica. Quando comparados com as outras formas sólidas utilizadas, os cocristais proporcionam uma maior diversidade de entidades possíveis de serem produzidas, permitem a utilização simultânea de vários grupos funcionais de um mesmo composto (incluindo aqueles que não são suficientemente ácidos ou básicos para a formação de sais, por exemplo), além de possibilitarem o desenho da estrutura de interesse e de serem inseridos em métodos de produção livres de solventes e que seguem a linha da química verde. Uma das principais vantagens dos cocristais é a formação de uma grande variedade de sólidos com propriedades físico-químicas diferentes dos componentes cristalinos individuais, as quais podem ser moduladas. Embora os cocristais ofereçam oportunidades de modificações das propriedades físico-químicas dos fármacos, como a solubilidade e a estabilidade, esta é uma área ainda pouco pesquisada no Brasil. Este artigo procurou abordar os diferentes aspectos relacionados aos cocristais, contribuindo, assim, para popularizar o conhecimento sobre o tema, permitindo determinar as relações entre a composição dos cocristais e as características físico-químicas que podem ser moduladas nestes sólidos. Ressalta-se que as pesquisas nesta área têm sido, cada vez mais, interdisciplinares, abrangendo estudos químicos, físico-químicos, farmacêuticos, toxicológicos, permitindo uma melhor compreensão dos mecanismos envolvidos no desempenho dos cocristais.

\section{AGRADECIMENTOS}

Os autores agradecem à CAPES (Projeto PVE A065_2013) e CNPq pelo apoio financeiro. G. S. Rauber agradece à CAPES e COTReino Unido pelo apoio financeiro (CsF BEX 9530/13-4).

\section{REFERÊNCIAS}

1. Schultheiss, N.; Newman, A.; Cryst. Growth Des. 2009, 9, 2950.

2. Aitipamula, S.; Banerjee, R.; Bansal, A. K.; Biradha, K.; Cheney, M. L.; Choudhury, A. R.; Desiraju, G. R.; Dikundwar, A. G.; Dubey, R.; Duggirala, N.; Ghogale, P. P.; Ghosh, S.; Goswami, P. K.; Goud, N. R.; Jetti, R.; Karpinski, P.; Kaushik, P.; Kumar, D.; Kumar, V.; Moulton, B.; Mukherjee, A.; Mukherjee, G.; Myerson, A. S.; Puri, V.; Ramanan, A.; Rajamannar, T.; Reddy, C. M.; Rodríguez-Hornedo, N.; Rogers, R. D.; Row, T. N. G.; Sanphui, P.; Shan, N.; Shete, G.; Singh, A.; Sun, C. Q. C.; Swift, J. A.; Thaimattam, R.; Thakur, T. S.; Thaper, R. K.; Thomas, S. P.; Tothadi, S.; Vangala, V. R.; Variankaval, N.; Vishweshwar, P.; Weyna, D. R.; Zaworotko, M. J.; Cryst. Growth Des. 2012, 12, 2147.

3. Blagden, N.; Colesb, S. J.; Berry, D. J.; CrystEngComm 2014, 16, 5753.

4. Good, D. J.; Rodríguez-Hornedo, N.; Cryst. Growth Des. 2009, 9, 2252.

5. McNamara, D. P.; Childs, S. L.; Giordano, J.; Iarriccio, A.; Cassidy, J.; Shet, M. S.; Mannion, R.; O’Donnell, E.; Park, A.; Pharm. Res. 2006, 23, 1888 .

6. Remenar, J. F.; Morissette, S. L.; Peterson, M. L.; Moulton, B.; MacPhee, J. M.; Guzman, H. R.; Almarsson, O.; J. Am. Chem. Soc. 2003, 125,8456 .

7. Hickey, M. B.; Peterson, M. L.; Scoppettuolo, L. A.; Morrisette, S. L.; Vetter, A.; Guzmán, H.; Remenar, J. F.; Zhang, Z.; Tawa, M. D.; Haley, S.; Zaworotko, M. J.; Almarsson, Ö.; Eur. J. Pharm. Biopharm. 2007, 67, 112.

8. Kuminek, G.; Cao, F.; Rocha, A. B. O.; Cardoso, S. G.; Rodríguez-Homedo, N.; Adv. Drug Deliver. Rev. 2016, 101, 143.

9. Roy, L.; Lipert, M. P.; Rodríguez-Hornedo, N. Em Pharmaceutical Salts and Co-crystals; Wouters, J.; Quére, L., eds.; Royal Society of Chemistry: London, 2011, cap. 11.

10. Bethune, S. J.; Huang, N.; Jayasankar, A.; Rodríguez-Hornedo, N.; Cryst. Growth. Des. 2009, 9, 3976.

11. Alhalaweh, A.; Roy, L.; Rodríguez-Hornedo, N.; Velaga, S. P.; Mol. Pharmaceutics 2012, 9, 2605.

12. Reddy, L. S.; Bethune, S. J.; Kampf, J. W.; Rodríguez-Hornedo, N.; Cryst. Growth. Des. 2009, 9, 378.

13. Kuminek, G.; Rodríguez-Homedo, N.; Siedler, S.; Rocha, H. V. A.; Cuffini, S. L.; Cardoso, S. G.; Chem. Commun. 2016, 52, 5832.

14. Huang, N.; Rodríguez-Hornedo, N.; Cryst. Growth Des. 2010, 10, 2050.

15. Huang, N.; Rodríguez-Hornedo, N.; J. Pharm. Sci. 2011, 100, 5219.

16. Lipert, M. P.; Rodríguez-Hornedo, N.; Mol. Pharmaceutics 2015, 12, 3535 . 
17. Lipert, M. P.; Roy, L.; Childs, S. L.; Rodríguez-Hornedo, N.; J. Pharm. Sci. 2015, 104, 4153 .

18. Good, D. J.; Rodríguez-Hornedo, N.; Cryst. Growth Des. 2009, 9, 2252.

19. Basavoju, S.; Boström, D.; Velaga, S.; Pharm. Res. 2008, 25, 530.

20. Desiraju, G. R.; J. Am. Chem. Soc. 2013, 135, 9952.

21. Desiraju, G. R. Em Pharmaceutical Salts and Co-crystals; Wouters, J.; Quére, L., eds.; Royal Society of Chemistry: London, 2011, cap. 1.

22. Allen, F. H.; Acta Crystallogr. B. 2002, 58, 380.

23. Wouters, J.; Rome, S.; Quere, L. Em Pharmaceutical Salts and Cocrystals; Wouters, J.; Quére, L., eds.; Royal Society of Chemistry: London, 2011, cap. 16.

24. Fábián, L.; Cryst. Growth Des. 2009, 9, 1436.

25. Desiraju, G. R.; Angew. Chem., Int. Ed. Engl. 1995, 34, 2311.

26. Desiraju, G. R.; Chem. Commun. 1997, 1475.

27. Almarsson, O.; Zaworotko, M. J.; Chem. Commun. 2004, 1889.

28. Johnson, S. L.; Rumon, K. A.; J. Phys. Chem. 1965, 69, 74.

29. Bond, A. D. Em Pharmaceutical Salts and Co-crystals; Wouters, J.; Quére, L., eds.; Royal Society of Chemistry: London, 2011, cap. 2.

30. Cherukuvada, S.; Nangia, A.; CrystEngComm 2012, 14, 7840.

31. Grobelny, P.; Mukherjee, A.; Desiraju, G. R.; CrystEngComm 2011, 13, 4358.

32. Price, S. L.; Chem. Soc. Rev. 2014, 43, 2098.

33. Gebauer, D.; Kellermeier, M.; Gale, J. D.; Bergstrom, L.; Colfen, H.; Chem. Soc. Rev. 2014, 43, 2348.

34. Myerson, A. S.; Trout, B. L.; Science 2013, 341, 855.

35. Bruno, I. J.; Cole, J. C.; Edgington, P. R.; Kessler, M.; Macrae, C. F.; McCabe, P.; Pearson, J.; Taylor, R.; Acta Crystallogr. B. 2002, 58, 389.

36. Macrae, C. F.; Bruno, I. J.; Chisholm, J. A.; Edgington, P. R.; McCabe, P.; Pidcock, E.; Rodríguez-Monge, L.; Taylor, R.; van de Streek, J.; Wood, P. A.; J. Appl. Crystallogr. 2008, 41, 466.

37. Yan, Y.; Chen, J. M.; Lu, T. B., CrystEngComm 2013, 15, 6457.

38. Stanton, C.; Mookadam, F.; Cha, S.; McNamara, D.; Aukrust, P.; Wojnicz, R.; Bailey, K. R.; Cooper, L. T.; Int. J. Cardiol. 2008, 128, 38.

39. Trask, A. V.; Motherwell, W. D. S.; Jones, W.; Cryst. Growth Des. 2005, 5, 1013.

40. Chieng, N.; Hubert, M.; Saville, D.; Rades, T.; Aaltonen, J.; Cryst. Growth Des. 2009, 9, 2377.

41. Martin, F. A.; Pop, M. M.; Borodi, G.; Filip, X.; Kacso, I.; Cryst. Growth Des. 2013, 13, 4295.

42. Shayanfar, A.; Jouyban, A.; Powder Technol. 2014, 262, 242.

43. Aitipamula, S.; Wong, A. B. H.; Chow, P. S.; Tan, R. B. H.; CrystEngComm 2012, 14, 8515.

44. Childs, S. L.; Chyall, L. J.; Dunlap, J. T.; Smolenskaya, V. N.; Stahly, B. C.; Stahly, G. P.; J. Am. Chem. Soc. 2004, 126, 1335.

45. Harriss, B. I.; Vella-Zarb, L.; Wilson, C.; Evans, I. R.; Cryst. Growth Des. 2014, 14, 783.

46. Ueto, T.; Takata, N.; Muroyama, N.; Nedu, A.; Sasaki, A.; Tanida, S.; Terada, K.; Cryst. Growth Des. 2012, 12, 485.

47. Goud, N. R.; Gangavaram, S.; Suresh, K.; Pal, S.; Manjunatha, S. G.; Nambiar, S.; Nangia, A.; J. Pharm. Sci. 2012, 101, 664.

48. Aitipamula, S.; Vangala, V. R.; Chow, P. S.; Tan, R. B. H.; Cryst. Growth Des. 2012, 12, 5858.

49. Sanphui, P.; Devi, V. K.; Clara, D.; Malviya, N.; Ganguly, S.; Desiraju, G. R.; Mol. Pharmaceutics 2015, 12, 1615.

50. Oberoi, L. M.; Alexander, K. S.; Riga, A. T.; J. Pharm. Sci. 2005, 94, 93.

51. Berry, D. J.; Seaton, C. C.; Clegg, W.; Harrington, R. W.; Coles, S. J.; Horton, P. N.; Hursthouse, M. B.; Storey, R.; Jones, W.; Friscic, T.; Blagden, N.; Cryst. Growth Des. 2008, 8, 1697.

52. Walsh, R. D. B.; Bradner, M. W.; Fleischman, S.; Morales, L. A.; Moulton, B.; Rodríguez-Hornedo, N.; Zaworotko, M. J.; Chem. Commun. 2003, 186.

53. Jung, M.-S.; Kim, J.-S.; Kim, M.-S.; Alhalaweh, A.; Cho, W.; Hwang, S.-J.; Velaga, S. P.; J. Pharm. Pharmacol. 2010, 62, 1560.
54. Aitipamula, S.; Wong, A. B. H.; Chow, P. S.; Tan, R. B. H.; CrystEngComm 2013, 15, 5877.

55. Cheney, M. L.; Weyna, D. R.; Shan, N.; Hanna, M.; Wojtas, L.; Zaworotko, M. J.; J. Pharm. Sci. 2011, 100, 2172.

56. Caira, M. R.; Bourne, S. A.; Samsodien, H.; Engel, E.; Liebenberg, W.; Stieger, N.; Aucamp, M.; CrystEngComm 2012, 14, 2541.

57. Cherukuvada, S.; Babu, N. J.; Nangia, A.; J. Pharm. Sci. 2011, 100, 3233.

58. Basavoju, S.; Bostrom, D.; Velaga, S. P.; Cryst. Growth Des. 2006, 6 , 2699.

59. Luo, Y. H.; Sun, B. W.; Cryst. Growth Des. 2013, 13, 2098.

60. Childs, S. L.; Hardcastle, K. I.; Cryst. Growth Des. 2007, 7, 1291.

61. Smith, A. J.; Kavuru, P.; Wojtas, L.; Zaworotko, M. J.; Shytle, R. D.; Mol. Pharmaceutics 2011, 8, 1867.

62. Saha, R.; Sengupta, S.; Dey, S. K.; Steele, I. M.; Bhattacharyya, A.; Biswas, S.; Kumar, S.; RSC Adv. 2014, 4, 49070.

63. Zegarac, M.; Leksic, E.; Sket, P.; Plavec, J.; Bogdanovic, M. D.; Bucar, D. K.; Dumic, M.; Mestrovic, E.; CrystEngComm 2014, 16, 32.

64. Chadha, R.; Bhandari, S.; Haneef, J.; Khullar, S.; Mandal, S.; CrystEngComm 2014, 16, 8375.

65. Babu, N. J.; Sanphui, P.; Nangia, A.; Chem. - Asian J. 2012, 7, 2274.

66. Amidon, G. L.; Lennernas, H.; Shah, V. P.; Crison, J. R.; Pharm. Res. 1995, 12, 413.

67. Chiarella, R. A.; Davey, R. J.; Peterson, M. L.; Cryst. Growth Des. 2007, 7,1223 .

68. Rodríguez-Hornedo, N.; Nehm, S. J.; Seefeldt, K. F.; Pagán-Torres, Y.; Falkiewicz, C. J.; Mol. Pharmaceutics 2006, 3, 362.

69. Childs, S. L.; Rodríguez-Hornedo, N.; Reddy, L. S.; Jayasankar, A.; Maheshwari, C.; McCausland, L.; Shipplett, R.; Stahly, B. C.; CrystEngComm 2008, 10, 856.

70. Delori, A.; Friscic, T.; Jones, W.; CrystEngComm 2012, 14, 2350.

71. Lu, E.; Rodríguez-Hornedo, N.; Suryanarayanan, R.; CrystEngComm 2008, 10, 665 .

72. Nehm, S. J.; Rodríguez-Spong, B.; Rodríguez-Hornedo, N.; Cryst. Growth Des. 2006, 6, 592.

73. Jayasankar, A.; Good, D. J.; Rodríguez-Hornedo, N.; Mol. Pharmaceutics 2007, 4, 360 .

74. Braga, D.; Giaffreda, S. L.; Grepioni, F.; Chierotti, M. R.; Gobetto, R.; Palladino, G.; Polito, M.; CrystEngComm 2007, 9, 879.

75. Braga, D.; Giaffreda, S. L.; Rubini, K.; Grepioni, F.; Chierotti, M. R.; Gobetto, R.; CrystEngComm 2007, 9, 39.

76. Etter, M. C.; Acc. Chem. Res. 1990, 23, 120.

77. Etter, M. C.; J. Phys. Chem. 1991, 95, 4601.

78. Friscic, T.; Childs, S. L.; Rizvi, S. A. A.; Jones, W.; CrystEngComm 2009, 11, 418.

79. Friscic, T.; Jones, W.; Cryst. Growth Des. 2009, 9, 1621.

80. Karki, S.; Friscic, T.; Jones, W.; CrystEngComm 2009, 11, 470.

81. Shan, N.; Toda, F.; Jones, W.; Chem. Commun. 2002, 2372.

82. Trask, A. V.; Motherwell, W. D. S.; Jones, W.; Chem. Commun. 2004, 890.

83. Jayasankar, A.; Somwangthanaroj, A.; Shao, Z. J.; Rodríguez-Hornedo, N.; Pharm. Res. 2006, 23, 2381.

84. Seefeldt, K.; Miller, J.; Alvarez-Nunez, F.; Rodríguez-Hornedo, N.; J. Pharm. Sci. 2007, 96, 1147.

85. Harris, K. D. M.; Tremayne, M.; Kariuki, B. M.; Angew. Chem., Int. Ed. Engl. 2001, 40, 1626.

86. Reutzel-Edens, S. M. Em Pharmaceutical Salts and Co-crystals; Wouters, J.; Quére, L., eds.; Royal Society of Chemistry: London, 2011, cap. 10

87. Giron, D.; J. Therm. Anal. Calorim. 2002, 68, 335.

88. Yu, L. A.; Acc. Chem. Res. 2010, 43, 1257.

89. Chadwick, K.; Davey, R.; Cross, W.; CrystEngComm 2007, 9, 732.

90. Alleso, M.; Velaga, S.; Alhalaweh, A.; Cornett, C.; Rasmussen, M. A.; 
van den Berg, F.; de Diego, H. L.; Rantanen, J.; Anal. Chem. 2008, 80 7755.

91. Bugay, D. E.; Pharm. Res. 1993, 10, 317.

92. Harris, R. K.; Solid State Sci. 2004, 6, 1025.

93. Vogt, F. G.; Clawson, J. S.; Strohmeier, M.; Edwards, A. J.; Pham, T. N.; Watson, S. A.; Cryst. Growth Des. 2009, 9, 921.

94. Umprayn, K.; Mendes, R. W.; Drug Dev. Ind. Pharm. 1987, 13, 653.

95. Zografi, G.; Drug Dev. Ind. Pharm. 1988, 14, 1905.

96. Brittain, H. G.; Bogdanowich, S. J.; Bugay, D. E.; Devincentis, J.; Lewen, G.; Newman, A. W.; Pharm. Res. 1991, 8, 963.

97. Newman, A. W.; Reutzel-Edens, S. M.; Zografi, G.; J. Pharm. Sci. 2008, 97, 1047.

98. Good, D. J.; Rodríguez-Hornedo, N.; Cryst. Growth Des. 2010, 10, 1028.

99. Huang, N.; Rodríguez-Hornedo, N.; CrystEngComm 2011, 13, 5409.

100. Thakuria, R.; Delori, A.; Jones, W.; Lipert, M. P.; Roy, L.; RodríguezHornedo, N.; Int. J. Pharm. 2013, 453, 101.

101. Maheshwari, C.; Andre, V.; Reddy, S.; Roy, L.; Duarte, T.; RodrigezHornedo, N.; Cryst EngComm 2012, 14, 4801.

102. Maheshwari, C.; Tese de doutorado, University of Michigan, USA, 2012.

103. Stahl, P. H.; Wermuth, C. G., Handbook of pharmaceutical salts : properties, selection, and use, $2^{\text {nd }}$ ed.; Wiley-VCH: Weinheim, 2011.

104. Avdeef, A.; Voloboy, D.; Foreman, A. Em Comprehensive Medicinal Chemistry II; Taylor, J.B.; Triggle, D. J., eds.; Elsevier: Oxford, 2007, cap.5.

105. Serajuddin, A. T.; Adv. Drug Deliv. Rev. 2007, 59, 603.

106. Roy, L.; Lipert, M. P.; Huang, N.; Rodríguez-Hornedo, N.; Resumo da AAPS Annual Meeting and Exposition, New Orleans, USA, 2010.
107. Roy, L.; Tese de doutorado, University of Michigan, USA, 2013.

108. Roy, L.; Rodríguez-Hornedo, N., Resumo da AAPS Annual Meeting and Exposition, New Orleans, USA, 2010.

109. Weyna, D. R.; Cheney, M. L.; Shan, N.; Hanna, M.; Zaworotko, M. J.; Sava, V.; Song, S.; Sanchez-Ramos, J. R.; Mol. Pharmaceutics 2012, 9 , 2094.

110. Cheney, M. L.; Shan, N.; Healey, E. R.; Hanna, M.; Wojtas, L.; Zaworotko, M. J.; Sava, V.; Song, S.; Sanchez-Ramos, J. R.; Cryst. Growth Des. 2010, 10, 394.

111. Galia, E.; Nicolaides, E.; Horter, D.; Lobenberg, R.; Reppas, C.; Dressman, J. B.; Pharm. Res. 1998, 15, 698.

112. Naylor, L. J.; Bakatselou, V.; RodríguezHornedo, N.; Weiner, N. D.; Dressman, J. B.; Eur. J. Pharm. Biopharm. 1995, 41, 346.

113. Charman, W. N.; Porter, C. J. H.; Mithani, S.; Dressman, J. B.; J. Pharm. Sci. 1997, 86, 269.

114. Bakatselou, V.; Oppenheim, R. C.; Dressman, J. B.; Pharm. Res. 1991, 8, 1461.

115. Miyazaki, S.; Yamahira, T.; Morimoto, Y.; Nadai, T.; Int. J. Pharm. 1981, $8,303$.

116. Lipert, M. P., Tese de doutorado, University of Michigan, USA, 2015.

117. Lipert, M. P.; Roy, L.; Rodríguez-Hornedo, N.; Resumo da AAPS Annual Meeting and Exposition, Chicago, USA, 2012.

118. Lipert, M. P.; Rodríguez-Hornedo, N.; Resumo da AAPS Annual Meeting and Exposition, San Diego, USA, 2014

119. Lipert, M. P.; Rodríguez-Hornedo, N.; Resumo da AAPS Annual Meeting and Exposition, San Antonio, USA, 2013. 